\title{
Impact of the Tropopause Temperature on the Intensity of Tropical Cyclones: An Idealized Study Using a Mesoscale Model
}

\author{
SHUGUANG WANG \\ Department of Applied Physics and Applied Mathematics, Columbia University, New York, New York \\ SUZANA J. CAMARGO \\ Lamont-Doherty Earth Observatory, Columbia University, Palisades, New York \\ Adam H. Sobel And Lorenzo M. Polvani \\ Department of Applied Physics and Applied Mathematics, and Department of Earth and Environmental Sciences, \\ Columbia University, New York, and Lamont-Doherty Earth Observatory, Columbia University, \\ Palisades, New York
}

(Manuscript received 21 January 2014, in final form 10 July 2014)

\begin{abstract}
This study investigates the impact of the tropopause temperature on the intensity of idealized tropical cyclones (TCs) superimposed on background states of radiative-convective equilibrium (RCE) in a threedimensional (3D) mesoscale model. Simulations are performed with constant sea surface temperature and an isothermal stratosphere with constant tropopause temperature. The potential intensity (PI) computed from the thermodynamic profiles of the RCE state (before the TCs are superimposed on it) increases by $0.4-1 \mathrm{~m} \mathrm{~s}^{-1}$ for each $1 \mathrm{~K}$ of tropopause temperature reduction. The 3D TC experiments yield intense tropical cyclones whose intensities exceed the PI value substantially. It is further shown that the discrepancy may be largely explained by the supergradient wind in the 3D simulations. The intensities of these 3D TCs increase by $\sim 0.4 \mathrm{~m} \mathrm{~s}^{-1}$ per $1 \mathrm{~K}$ of cooling in the tropopause temperature in RCE, on the low end of the PI dependence on the tropopause temperature. Sensitivity experiments with a larger horizontal grid spacing of $8 \mathrm{~km}$ produce less intense TCs, as expected, but similar dependence $\left(\sim-0.5 \mathrm{~m} \mathrm{~s}^{-1} \mathrm{~K}^{-1}\right)$ on tropopause temperature. Equilibrium TC solutions are further obtained in 200-day experiments with different values of constant stratospheric temperature. Similar relationships between TC intensity and tropopause temperature are also found in these equilibrium TC solutions.
\end{abstract}

\section{Introduction}

The cooling trend in the tropical lower stratosphere is one of the most dramatic climate change signals in the recent climate record (Randel et al. 2009; Emanuel et al. 2013; Vecchi et al. 2013). Recent studies (Emanuel 2010; Emanuel et al. 2013; Vecchi et al. 2013) have shown evidence that this stratospheric temperature trend near the tropopause may have influenced tropical cyclone (TC) activity in the tropical North Atlantic basin. These recent results provide motivation to study the influence

Corresponding author address: Shuguang Wang, Department of Applied Physics and Applied Mathematics, Columbia University, 500 W. 120th St., S. W. Mudd, Rm. 290, New York, NY 10027.

E-mail: sw2526@columbia.edu of thermodynamic conditions in the upper troposphere and lower stratosphere on TCs in more detail.

Potential intensity theory (Emanuel 1987) models TCs as heat engines, operating between the warm ocean and the cold tropopause and converting thermal energy to mechanical energy. Variations in the sea surface temperature (SST) have received much attention in the literature (e.g., Vecchi and Soden 2007; Ramsay and Sobel 2011). The effect of temperature near the tropopause has been less thoroughly studied. It is often assumed that the TC outflow temperature is directly set by the ambient tropopause temperature via gravity wave adjustment. Recent developments in TC theory (Emanuel and Rotunno 2011; Emanuel 2012), however, suggest that this conventional view of the TC outflow is incomplete. These authors hypothesized that the criticality 
of the Richardson number $R_{i}$ in the outflow imposes a strong constraint on the stratification of the outflow temperature, which sets the radial distribution of the wind speed through moist adjustment.

Emanuel (2010) and Emanuel et al. (2013) provided observational evidence that temperature variations in the stratosphere have contributed to the interannual variability of the TC potential intensity in the last 30 years. Yet, because of well-known observational issues, tropical temperatures in the upper troposphere and lower stratosphere in the reanalysis datasets [e.g., European Centre for Medium-Range Weather Forecasts (ECMWF) Re-Analysis (ERA) and National Centers for Environmental Prediction-National Center for Atmospheric Research (NCEP-NCAR) Global Reanalysis 1 (NCEP-1; Kalnay et al. 1996)] are uncertain (Vecchi et al. 2013). Perhaps equally importantly, the impact of temperature at these levels on TC intensity is difficult to assess from observations.

Another line of evidence for the importance of the tropopause temperature comes from global climate models (Vecchi et al. 2013) that can faithfully simulate interannual variability of TC activity (Zhao et al. 2009; Zhao and Held 2012) as well as regional downscaling systems designed for similar objectives (Knutson et al. 2008; Hill and Lackmann 2011). Vecchi et al. (2013) also showed that the North Atlantic TC activity simulated by a high-resolution climate model and a regional TC downscaling system is modulated by changes in temperature in the upper troposphere/lower stratosphere (UT/LS) region, although the effect is much weaker than in Emanuel's studies (Emanuel 2010; Emanuel et al. 2013).

Several axisymmetric modeling studies have documented the effect of the outflow temperature on simulated TC intensity (Rotunno and Emanuel 1987; Mrowiec et al. 2011; Emanuel and Rotunno 2011; Ramsay 2013; Chavas and Emanuel 2014). Ramsay (2013) compared the effect of the tropopause temperature to SST in an idealized axisymmetric model and found that $1-\mathrm{K}$ warming in SST can change the potential intensity (PI) by $2 \mathrm{~m} \mathrm{~s}^{-1}$ while a 1-K decrease in the tropopause temperature increases PI by $1 \mathrm{~m} \mathrm{~s}^{-1}$. Because the UT/LS temperature has larger natural variability than SST at seasonal, interannual, and decadal time scales, and the latter is ultimately limited by the thermal inertia of the ocean, this suggests that, as also noted in Emanuel (2010), the tropopause temperature may have played a more important role than SST in the recent interannual variability of TC activity.

While much about TC-environment interaction has been learned using axisymmetric models (e.g., Ooyama 1969; Rotunno and Emanuel 1987; Craig and Gray 1996; Persing and Montgomery 2003; Hausman et al. 2006; Bryan and Rotunno 2009a,b; Mrowiec et al. 2011; Hakim
2011; Emanuel and Rotonno 2011; Chavas and Emanuel 2014), several recent studies (e.g., Yang et al. 2007; Persing et al. 2013) have demonstrated that ignoring asymmetric dynamics may lead to biased estimates of TC intensity, suggesting that explicitly resolved threedimensional (3D) TC structure and convective eddies may be important for studying TC-environment interaction. In this work, we use a $3 \mathrm{D}$, full-physics mesoscale model to 1) examine the effect of the tropopause temperature on hurricane intensity and 2) further compare the predictions of potential intensity theory to the results of 3D modeling experiments.

The rest of this paper is organized as follows. Section 2 contains a description of the numerical experiments, followed by the results of transient TC solutions in section 3 and equilibrium TC solutions in section 4 . Section 5 summarizes the results.

\section{Experiment design}

We perform numerical simulations of idealized tropical cyclones developed from a finite amplitude vortex. The initial vortex is the same as that specified in Rotunno and Emanuel (1987). This is an idealized moist warm core vortex in gradient and hydrostatic balance, with the maximum wind speed at the surface. The warm-core structure differs from the observed cold cores typically found during early phases of TC genesis (Bister and Emanuel 1997; Raymond et al. 2007). Because of this, the subsequent development of the initial vortex does not represent the transition from the cold to warm core, as in some other studies (e.g., Montgomery et al. 2006; Rappin et al. 2010). Nevertheless, this warm-core vortex initialization suffices for our purpose, since the present study focuses on the intensity of the mature TC.

The environment into which the TC vortex is introduced has a horizontal mean taken from a radiative-convective equilibrium (RCE) simulation in a small domain, similar to the procedure in Nolan et al. (2007). The RCE soundings do not represent any specific region of the real atmosphere of Earth or any tropical ocean basin. A distinct advantage of using an RCE sounding is that the environment is already close to equilibrated before the TC is introduced. While the presence of the TC itself can change the mean state, the effect of any initial shock from disequilibrium in the environment itself (apart from the TC's own influence) on the TC solution is small. This is desirable in a study of the influence of the environment on TC intensity.

The Advanced Research Weather Research and Forecasting (WRF) Model, version 3.0 (Skamarock et al. 2008), is used for this study. The radiation package is replaced by a Newtonian cooling scheme (Pauluis and Garner 2006; Wang and Sobel 2011): 
TABLE 1. Three-dimensional RCE and TC experiments.

\begin{tabular}{lc}
\hline \hline RCE & $T_{o}=185,190, \ldots, 225 \mathrm{~K} ; \mathrm{SST}=28^{\circ} \mathrm{C} ;$ \\
& $D x=4 \mathrm{~km}$ \\
& $T_{o}=195,200,205,210,215 \mathrm{~K} ;$ \\
& $\mathrm{SST}=26^{\circ}$ and $30^{\circ} \mathrm{C} ; D x=4 \mathrm{~km}$ \\
& $T_{o}=195,200,205,210,215 \mathrm{~K} ;$ \\
& $\mathrm{SST}=28^{\circ} \mathrm{C} ; D x=8 \mathrm{~km}$ \\
& Small domain $(200 \mathrm{~km})$ \\
& $T_{o}=195,200,205,210,215 \mathrm{~K} ;$ \\
& $\mathrm{SST}=26^{\circ}, 28^{\circ}$, and $30^{\circ} \mathrm{C} ;$ \\
& $D x=4 \mathrm{~km}$ \\
Transient TC & $T_{o}=195,200,205,210,215 \mathrm{~K} ;$ \\
& $\mathrm{SST}=28^{\circ} \mathrm{C} ; D x=8 \mathrm{~km}$ \\
& Two nest grids: $6000 \mathrm{~km}$ for the outer domain and $1200 \mathrm{~km}$ for the inner \\
domain & \\
& $T_{o}=195,200,205,210,215 \mathrm{~K} ;$ \\
SST $=28^{\circ} \mathrm{C} ; D x=8 \mathrm{~km}$ \\
Single domain $(3000 \mathrm{~km})$ \\
\end{tabular}

$\left.Q\right|_{\text {radiative cooling }}=\left\{\begin{array}{lll}-1.2 \mathrm{~K} \mathrm{day}^{-1} & \text { if } & T>T_{o}+6 \mathrm{~K} \\ -\frac{T-T_{o}}{5 \text { days }^{2}} & \text { if } & T \leq T_{o}+6 \mathrm{~K}\end{array}\right.$.

With this simple treatment of radiation, the troposphere is cooled at a constant rate of $1.2 \mathrm{~K} \mathrm{day}^{-1}$, which is close to the observed climatology of net radiative cooling, and stratospheric temperature is relaxed to a constant $T_{o}$. Hence, the stratosphere is isothermal and the tropopause temperature is approximately $T_{o}$. The parameter $T_{o}$ is used as the control for the numerical experiments in this study. The 6-K increment in Eq. (1) is included to make the heating rate continuous across the tropopause.

The RCE is computed on a doubly periodic numerical domain of $200 \times 200 \times 26 \mathrm{~km}^{3}$ at horizontal grid spacing $D x=4 \mathrm{~km}$ with a given a SST and $T_{o}$. The Coriolis parameter $f=0.5 \times 10^{-4} \mathrm{~s}^{-1}$. Using the RCE solution to define the initial state, we then perform two types of TC simulations: short-term ( $\sim 10$ days) simulations designed to identify the peak TC intensity obtained in a given environment and long-term ( $\sim 200$ days $)$ ones to obtain equilibrium TC solutions. Table 1 summarizes all the numerical experiments. Two computational domains are used in the transient TC integrations: a large one $\left(6000 \times 6000 \times 26 \mathrm{~km}^{3}\right)$, with $D x=12 \mathrm{~km}$; and a smaller one $\left(1200 \times 1200 \times 26 \mathrm{~km}^{3}\right)$ in the middle of the large one, with $D x=4 \mathrm{~km}$. Doubly periodic boundary conditions are adopted for the outer domain. We use 50 vertical levels with stretched vertical-level spacing. The computational domain in the equilibrium TC integrations uses only one grid $\left(3200 \times 3200 \times 26 \mathrm{~km}^{3}\right)$ with $D x=8 \mathrm{~km}$. The nominal model top of all numerical grids is set to $26 \mathrm{~km}$.

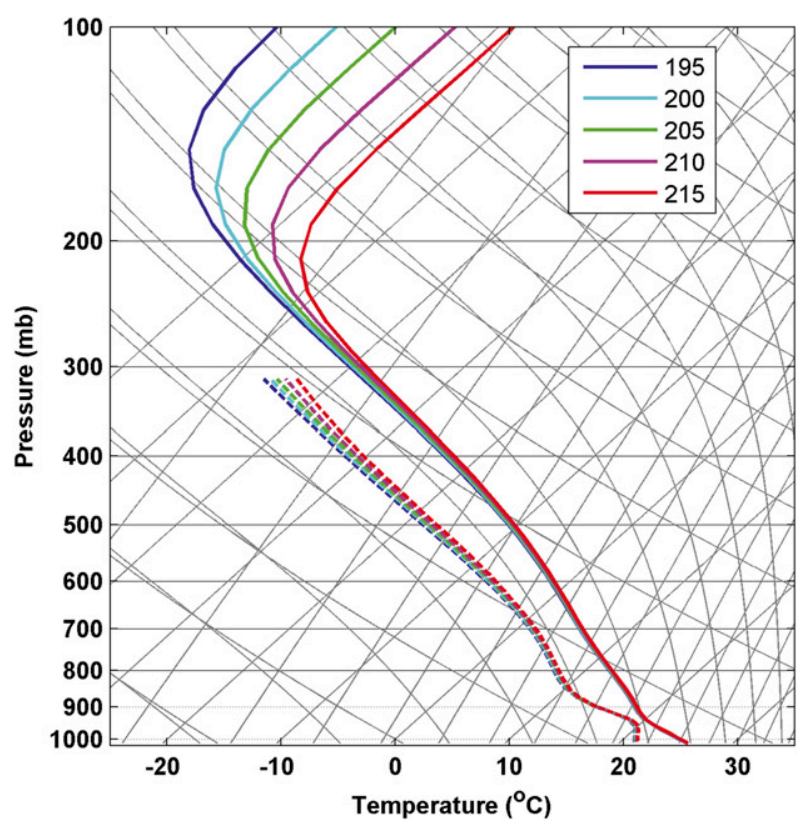

FIG. 1. Skew $T-\log p$ diagram of the RCE soundings with five values of $T_{o}(195,200,205,210$, and $215 \mathrm{~K})$ at SST $=28^{\circ} \mathrm{C}$.

We vary the tropopause temperature $T_{o}$ in Eq. (1) from 195 to $215 \mathrm{~K}$ in increments of $5 \mathrm{~K}$. This range is larger than that derived from either the NCEP-1 or 40-yr ECMWF Re-Analysis (ERA-40) datasets from the late 1970s to 2002, but it is intended to bracket the observationally derived range. The mean soundings from the RCE integrations with these $T_{o}$ values and SST set to $28^{\circ} \mathrm{C}$ are shown in Fig. 1. The largest difference in temperature among these soundings occurs at the tropopause level and the stratosphere, and the difference becomes smaller in the lower troposphere for both temperature and humidity.

Besides the simple treatment of radiative cooling, the following physics schemes are used for both the smalldomain RCE and large-domain TC integrations. Boundary layer turbulence and vertical transport by subgrid eddies are parameterized using the Yonsei University (YSU) scheme (Hong et al. 2006); horizontal transport by subgrid eddies is treated using the Smagorinsky firstorder closure; the surface moisture and heat fluxes are parameterized following the Monin-Obukhov similarity theory, together with the Charnock relationship for the surface roughness; and the Purdue-Lin scheme is used for cloud microphysics (Lin et al. 1983; Chen and Sun 2002). The horizontal and vertical advection schemes are fifth- and third-order accurate, respectively. Moisture and condensate are advected using a positive definite scheme. We use the implicit damping scheme to suppress unphysical reflection of vertically propagating gravity waves in the top $5 \mathrm{~km}$ of the numerical grid 
(Klemp et al. 2008). We adopted these physical schemes partly because we have found that both domain-averaged moisture and moist static energy are better conserved with these particular parameterization choices than with others (not shown). Convective parameterization is not used in any numerical experiment in this study.

The following paragraph contains some technical details that have significant implications for our TC integrations, but may be skipped for uninterested readers. The YSU boundary layer scheme parameterizes not only boundary layer eddies but also free-tropospheric turbulent eddies, including turbulence onset near the TC outflow regions. Prior studies by Nolan et al. (2009a,b) and Kepert (2012) showed that this scheme works reasonably well in the hurricane boundary layer despite its simplicity. Vertical eddy viscosities above the entrainment zone overlaying the mixed layer have a specified dependence on stability and mixing length. The latter varies with height, approaching $30 \mathrm{~m}$ in the free troposphere. Horizontal subgrid-scale eddy viscosity is a function of two environmental parameters: horizontal mixing length and flow deformation. The horizontal eddy viscosity coefficient is written as $K_{h}=[\operatorname{Cs}(D x)]^{2} S$, where $S$ is flow deformation and $C s$ is a constant $(0.25)$. Thus, the horizontal mixing length is resolution-dependent $l_{h}=\operatorname{Cs}(D x)$, and $l_{h}$ is $1 \mathrm{~km}$ in the $D x=4 \mathrm{~km}$ integration and $2 \mathrm{~km}$ in the $D x=8 \mathrm{~km}$ integration. Bryan and Rotunno (2009a,b) have examined the importance of $l_{h}$ for the TC intensity in axisymmetric models and have shown that asymmetries and turbulent eddies parameterized by mixing length may smooth out the frontal structure near the eyewall region, thus reducing the TC intensity. The vertical eddy viscosity in the free troposphere is a function of the vertical mixing length $l_{v}(30 \mathrm{~m})$, the stability function, and vertical wind shear. Onset of turbulence occurs when the local gradient Richardson number becomes negative. Dissipative heating is not included in this study. Tests with the dissipative heating effect (Bister and Emanuel 1998; Zhang and Altshuler 1999) reveal insignificant changes in the TC intensity in our model. While Bister and Emanuel (1998) and other subsequent numerical studies have suggested that the dissipation of kinetic energy at the air-sea interface through molecular diffusion may boost the TC intensity, a recent observational study by Zhang (2010) suggested that the effect of dissipative heating may be smaller by a factor of 3 than the formulation in Bister and Emanuel (1998). We do not pursue this issue further in this study.

Because of their highly axisymmetric structure, TCs are most conveniently studied in cylindrical coordinates. We use the following procedures to convert the 3D model fields from the Cartesian to cylindrical coordinates: 1) find the maximum and minimum values of surface pressure and calculate contours of surface pressure whose values lie within $30 \%$ and $70 \%$ of the range of the surface pressure, 2) fit each of the contours of surface pressure to an ellipse using least squares and use the geometric center of these ellipses as the origin of the cylindrical coordinates, and 3) interpolate 3D fields from Cartesian to cylindrical coordinates. This method yields higher maximum axisymmetric wind speeds by as much as $30 \%$ compared to alternative methods using either minimum sea level pressure or maximum vorticity as the origin, which may reduce maximum azimuthal wind speed due to aliasing of lowwavenumber features.

\section{Results}

\section{a. PI from RCE}

The PI expressed as the maximum wind speed $V$ $\left(\mathrm{m} \mathrm{s}^{-1}\right)$ can be written as

$$
V^{2}=\frac{C_{k}}{C_{d}} \frac{T^{s}-T^{o}}{T^{o}}\left(k^{*}-k\right),
$$

where $C_{k}$ and $C_{d}$ are the heat exchange and the drag coefficient, respectively; $T^{o}$ is outflow temperature [this differs from $T_{o}$, which is the relaxation constant in Eq. (1), and the approximate temperature of the idealized isothermal stratosphere]; $T^{s}$ is sea surface temperature; $k^{*}$ is the ocean surface enthalpy; and $k$ is the nearsurface air enthalpy. This expression states that PI should be a nonlinear function of the outflow temperature. A more accurate expression for PI (Bister and Emanuel 2002), derived from energy balance within a control volume along the angular momentum surface at the radius of maximum wind (RMW) is used in Emanuel and Bister's (2002) PI code to compute PI from an environmental sounding:

$$
V^{2}=\left.\frac{C_{k}}{C_{d}} \frac{T^{s}}{T^{o}}\left(\mathrm{CAPE}^{*}-\mathrm{CAPE}\right)\right|_{M},
$$

where CAPE is the convective available potential energy of a near-surface air parcel in the environmental sounding, while CAPE* is the same quantity except for a saturated air parcel lifted from sea surface at the RMW. The outflow temperature is the temperature at the level of neutral buoyancy for the rising parcel. Both CAPE and $T^{o}$ depend on specific thermodynamic assumptions. Uncertainties in several parameters may change the estimate of PI and its dependence on environmental parameters: the value of $C_{k} / C_{d}$, the thermodynamic assumptions used to compute CAPE, and the use of dissipative heating. 
(a) PI versus outflow temperature, $C_{k} / C_{d}=0.5$

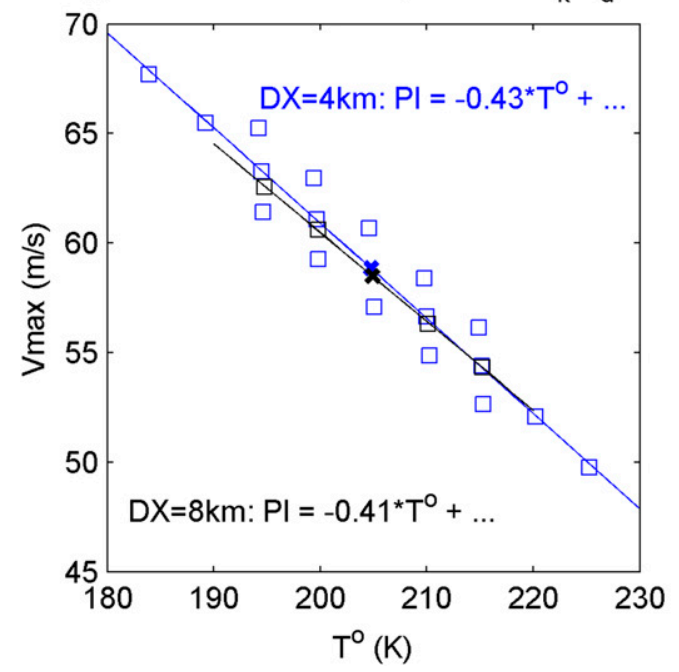

(b) PI versus outflow temperature, $C_{k} / C_{d}=0.9$

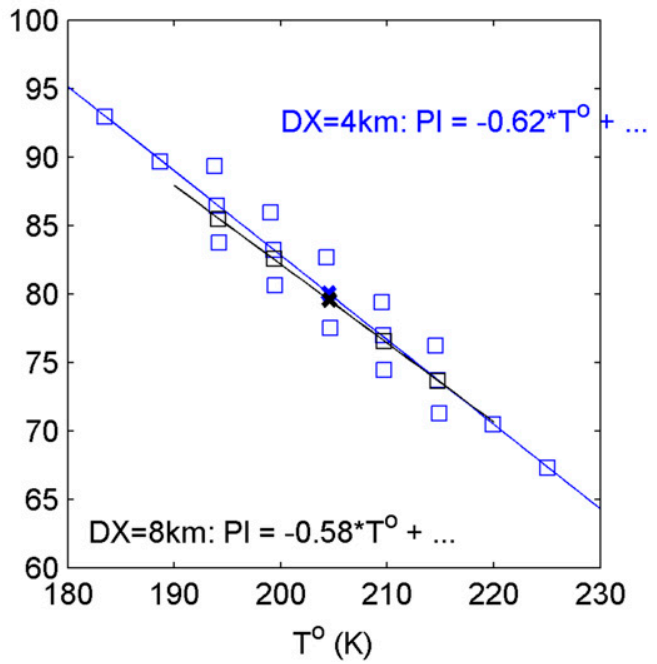

FIG. 2. PI as a function of $T^{o}$ computed from Emanuel's PI code. Blue squares denote those from 4-km smalldomain RCE integrations with SST $=26^{\circ}, 28^{\circ}$, and $30^{\circ} \mathrm{C}$; black squares denote those from 8-km small-domain RCE integrations with SST $=28^{\circ} \mathrm{C}$. Boldface crosses indicate results from the RCE sounding with SST $=28^{\circ} \mathrm{C}$ and $T_{o}=205 \mathrm{~K}$.

Figure 2 shows that the PI values computed from the RCE soundings vary approximately linearly as a function of outflow temperature $T^{o}$, at least in the limited range of $T_{o}$ studied. The outflow temperature (the $x$ axis) obtained from Emanuel's PI code corresponds well to $T_{o}$ in Eq. (1).

We use two different values of $C_{k} / C_{d}, 0.5$ and 0.9 , in the PI code for Figs. $2 \mathrm{a}$ and $2 \mathrm{~b}$, respectively. In our 3D WRF integrations, $C_{k}$ and $C_{d}$ are computed interactively within the model using the Charnock relation in the surface layer parameterization. The computed ratio is $\sim 0.9$ far from the radius of maximum wind and $\sim 0.4$ near RMW. The lower value, 0.4 , is yet smaller than the lower bound obtained from observation-based estimates (0.5-0.7) in several recent studies (Black et al. 2007; Haus et al. 2010; Bell et al. 2012; Zhang 2010). Strictly, the derivation of PI is consistent with the use of the value at the RMW, rather than the (here much larger) environmental value. However, in many applications-such as the computation of PI from large-scale observational datasets in situations where no TC exists-it is common to use environmental values. Here, we find that the actual values from the RMW give PI values that significantly underestimate the actual peak TC intensities produced by the model, while the environmental values give much better agreement with the simulated TC intensities.

We also consider three choices that differ from the default settings in the PI code. First, the effect of dissipative heating is switched off, because it is not used in our numerical WRF experiments. Second, pseudoadiabatic rather than reversible adiabatic thermodynamics is used in the computations of CAPE and outflow temperature. The former is more appropriate in the context of the PI theory, as discussed in Bryan and Rotunno (2009a). Finally, the reduction factor of surface maximum wind is set to 1 instead of the default value of 0.7 , since we compare directly to the maximum wind speed above the boundary layer.

Varying these PI parameters yields different PI estimates and, consequently, different relationships between PI and outflow temperature in the RCE soundings. Table 2 summarizes the slope from the linear regression between the PI values and $T_{o}$ for all the RCE soundings, as we use three values of $C_{k} / C_{d}(0.5,0.7$, and 0.9$)$, either pseudoadiabatic or reversible thermodynamics, and either dissipative heating or no dissipative heating. The PI value computed from an RCE sounding with SST = $28^{\circ} \mathrm{C}$ and $T_{o}=205 \mathrm{~K}$ is also shown in parentheses in Table 2. Because of uncertainties in several adjustable parameters in the formulation of the PI code as discussed above, the estimated PI dependence on the outflow temperature varies considerably. For example, using reversible thermodynamics and dissipative heating yields a $\sim 1 \mathrm{~m} \mathrm{~s}^{-1}$ increase of PI with $1 \mathrm{~K}$ cooling at the tropopause. Thus, for this plausible set of parameters, we estimate a range of PI dependence on the tropopause temperature between -1 and $-0.4 \mathrm{~m} \mathrm{~s}^{-1} \mathrm{~K}^{-1}$. We will show that the dependence of the modelsimulated actual TC intensity on the tropopause temperature from the $3 \mathrm{D}$ experiments of TCs is on the lower end of this estimate. 
TABLE 2. Dependence of PI on the outflow temperature with different of choices of thermodynamics, $C_{k} / C_{d}$, and dissipative heating, computed from the small-domain RCE soundings. The number in parentheses is the PI value for the case $\mathrm{SST}=28^{\circ} \mathrm{C}$ and $T_{o}=205 \mathrm{~K}$. Units for the two numbers are $\mathrm{m} \mathrm{s}^{-1} \mathrm{~K}^{-1}$ and $\mathrm{m} \mathrm{s}^{-1}$, respectively. Boldface values are the cases in Fig. 2.

\begin{tabular}{|c|c|c|c|c|}
\hline \multirow[b]{2}{*}{$C_{k} / C_{d}$} & \multicolumn{2}{|c|}{ Reversible thermodynamics } & \multicolumn{2}{|c|}{ Pseudoadiabatic thermodynamics } \\
\hline & Dissipative heating off & Dissipative heating on & Dissipative heating off & Dissipative heating on \\
\hline 0.5 & $-0.41(54)$ & $-0.67(66)$ & $-0.43(59)$ & $-0.73(72)$ \\
\hline 0.7 & $-0.49(64)$ & $-0.82(78)$ & $-0.53(70)$ & $-0.91(86)$ \\
\hline 0.9 & $-0.57(73)$ & $-0.96(89)$ & $-0.62(80)$ & $-1.07(99)$ \\
\hline
\end{tabular}

The above discussion of PI uses the 49 levels of the RCE soundings at which thermodynamic variables are defined. This differs from typical applications of PI in diagnosing climate models, which typically use about 13 pressure levels. We have computed PI at the standard pressure levels and found that the absolute PI values increase less than $5 \mathrm{~m} \mathrm{~s}^{-1}$, but the slope remains very similar (not shown). Thus, our PI computation is not sensitive to the number of vertical levels.

So far, the discussion has been centered on the PI code calculations for the estimate of TC intensity and its dependence on the outflow temperature. Rather than using the PI code itself, one can also derive a bulk estimate of the PI dependence on the outflow temperature without complications of estimating $C_{k} / C_{d}$ or making the thermodynamic assumptions in the PI code (K. A. Emanuel 2014, personal communication). Taking partial derivative of Eq. (2) with respect to $T^{o}$ while holding other parameters fixed, we have

$$
\frac{\partial V}{\partial T^{o}}=\frac{-V T^{s}}{2 T^{o}\left(T^{s}-T^{o}\right)} .
$$

The values of these variables in Eq. (4) can be estimated as $T^{s}=300 \mathrm{~K}, T^{o}=200 \mathrm{~K}$, and $V \sim 80 \mathrm{~m} \mathrm{~s}^{-1}$ (this is the maximum gradient wind in the experiment with SST $=$ $28^{\circ} \mathrm{C}$ and $T^{o}=205 \mathrm{~K}$; see the discussion below). This gives $\sim-0.6 \mathrm{~m} \mathrm{~s}^{-1} \mathrm{~K}^{-1}$. If the effect of dissipative heating is suppressed by a factor $T^{o} / T^{s}$ on the right-hand side of Eq. (2), taking partial derivative with respect to $T^{o}$ yields

$$
\frac{\partial V}{\partial T^{o}}=\frac{-V}{2\left(T^{s}-T^{o}\right)} .
$$

Using the above estimated values again gives $\sim-0.4 \mathrm{~m} \mathrm{~s}^{-1} \mathrm{~K}^{-1}$. Thus, the effect of dissipative heating increases the sensitivity of PI to the outflow temperature slightly. While Eqs. (4) and (5) are useful for giving bulk estimate, it is also important to bear in mind that they cannot replace the PI code since they require a prior knowledge of the maximum wind speed, which we obtained from the 3D simulations. If the PI code is used to estimate $V$, it is again subject to uncertainties in several key parameters that are not well constrained by existing observations.

\section{b. TC in an RCE environment}

\section{1) Structure of Simulated TCS}

In this section, we focus on the temporal and spatial structure of the TCs simulated in the RCE environment using the WRF Model. Figure 3 shows the time-radius plot of tangential wind speed and vertical velocity of the TC simulated with SST $=28^{\circ} \mathrm{C}$ and $T_{o}=205 \mathrm{~K}$. The tangential wind shows that the TC spins up and reaches over $95 \mathrm{~m} \mathrm{~s}^{-1}$ after day 3.75 , maintains its peak intensity for 1-1.5 days, and decays after day 5 . The peak intensity of this transient TC solution greatly exceeds the theoretical maximum wind speed calculated from the PI code, $\sim 59 \mathrm{~m} \mathrm{~s}^{-1}$, if $C_{k} / C_{d}=\sim 0.5$ at RMW is used, and even significantly exceeds the value of $\sim 80 \mathrm{~m} \mathrm{~s}^{-1}$ obtained if the environmental value, $C_{k} / C_{d}=\sim 0.9$ is used (see Fig. 2, Table 2).

Figure 4 displays the azimuthally averaged structure of this TC at its peak intensity. The temperature anomalies maximize in the upper troposphere around $12 \mathrm{~km}$, while cold temperature anomalies due to evaporation and melting are found near the surface between the 25- and 50-km radii. Positive moisture anomalies appear near the RMW because of moisture transport by the strong vertical motion (Fig. 4b). Negative moisture anomalies are seen in the lower free troposphere $150 \mathrm{~km}$ away from the center, primarily due to advective drying by the descending branch of the secondary flow. Boundary layer inflow (Fig. 4d) reaches $35 \mathrm{~m} \mathrm{~s}^{-1}$ and outflow in the upper troposphere reaches over $25 \mathrm{~m} \mathrm{~s}^{-1}$. A secondary outflow just above the boundary layer but below the melting layer is evident near the RMW. The azimuthalmean vertical velocity reaches $\sim 6 \mathrm{~m} \mathrm{~s}^{-1}$ near the RMW, indicating intense convective activity.

Emanuel and Rotunno (2011) showed that the Richardson number reaches a critical value in the outflow region, where the flow is marginally stable; moist neutrality further ensures that this criticality in the upper layer is communicated to the lower levels and sets the radial distribution of the azimuthal wind. Figure $4 \mathrm{e}$ 
(a) Tangential velocity $(\mathrm{m} / \mathrm{s})$

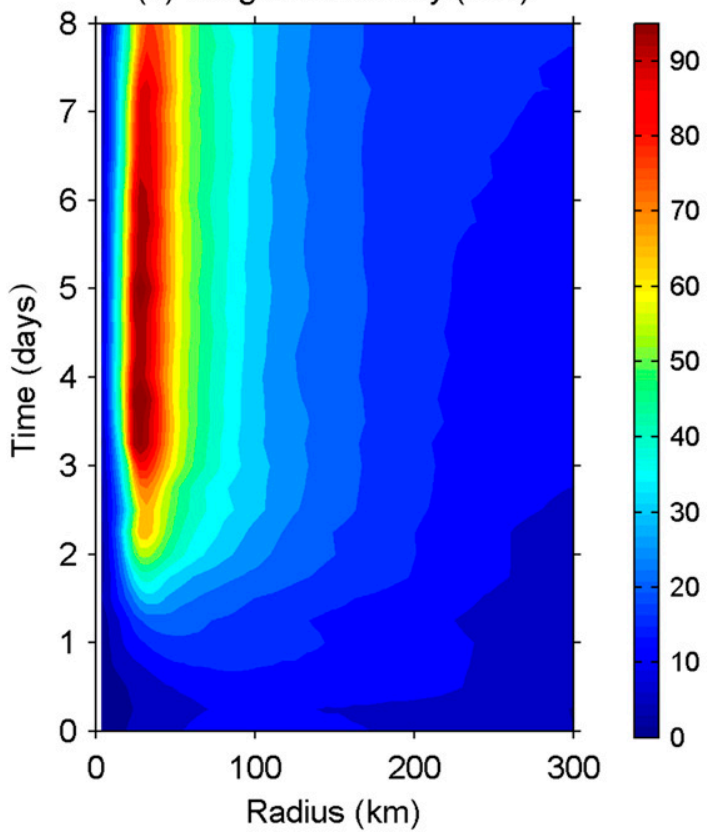

(b) Vertical velocity $(\mathrm{m} / \mathrm{s})$

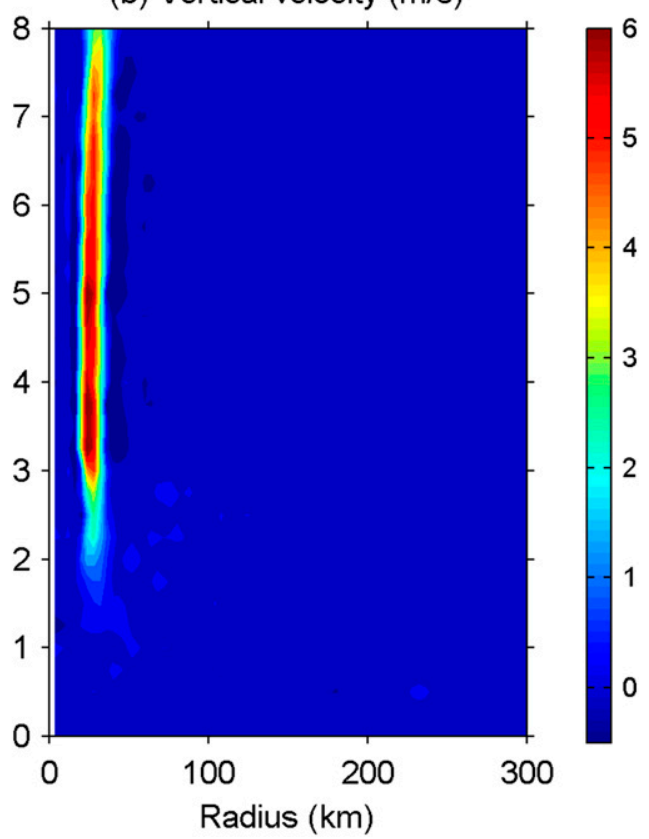

FIG. 3. Time-radius diagram of (a) tangential wind speed $\left(\mathrm{m} \mathrm{s}^{-1}\right)$ and (b) vertical velocity $\left(\mathrm{m} \mathrm{s}^{-1}\right)$ at the level of max wind speed $(\sim 1.3 \mathrm{~km})$ from the TC integration with SST $=28^{\circ} \mathrm{C}$ and $T_{o}=205 \mathrm{~K}$.

shows $R_{i}$ computed using the azimuthally averaged flow, where $R_{i}=N_{m}^{2} /\left(u_{z}^{2}+v_{z}^{2}\right)$, where $u_{z}$ and $v_{z}$ are the vertical wind shear of horizontal winds $u$ and $v$, respectively; and $N_{m}$ is the moist Brunt-Väisälä frequency. Note that the spatial structure of $R_{i}$ discussed below remains robust if $R_{i}$ is computed using 3D model output first and averaged in the azimuthal direction later. $R_{i}$ is less than zero over large regions below $7 \mathrm{~km}$ because the moist atmosphere is conditionally unstable.

We now focus on $R_{i}$ in the upper troposphere, where $R_{i}$ is less than one in three distinct layers with strong wind shear: immediately above the outflow jet $(\sim 14 \mathrm{~km})$ and below the tropopause, below the outflow jet $(\sim 12 \mathrm{~km})$, and below the return inflow $(\sim 9-10 \mathrm{~km})$. These three layers extend from $50 \mathrm{~km}$ to more than $250 \mathrm{~km}$ in radius. The value of $R_{i}$ is even closer to zero in the region between 50- and $100-\mathrm{km}$ radii and 9 and $11 \mathrm{~km}$ in height. The small value of $R_{i}$ in this area indicates the onset of moist turbulence, which is parameterized in the numerical model. This region of near-zero $R_{i}$ is in good agreement with Emanuel and Rotunno (2011, their Fig. 6a). The low Richardson number agrees with the observational study by Molinari et al. (2014), who found low $R_{i}$ using a large collection of the dropsondes.

The maximum azimuthal wind, $\sim 99 \mathrm{~m} \mathrm{~s}^{-1}$, is located at the top of the boundary layer (the black curves in Figs. $4 \mathrm{c}, \mathrm{d}$ ) at $\sim 1.3 \mathrm{~km}$. The boundary layer height is diagnosed in the YSU boundary scheme as the level at which the bulk Richardson number reaches its critical value. In the simulations, it covaries with the inflow depth in the radial direction. This differs from the boundary layer of a theoretical dry vortex, which decreases monotonically from the far field to the TC eye (Kepert 2001; Smith and Vogl 2008). The boundary layer height in Fig. $4 \mathrm{~d}$ also differs from the recent observational study in Zhang et al. (2011). We speculate that this discrepancy may be due to the fact that 1 ) the effect of moisture is not considered in the theoretical dry vortex and 2) the observed TCs are rarely as strong as those simulated in our numerical study.

\section{2) Simulated TC INTENSITY AND POTENTIAL INTENSITY}

PI theory (Emanuel 1987) plays an important role in studies of the hurricane-climate connection. It has been tested, to some extent, against observations (Emanuel 2000; Wing et al. 2007; Kossin and Camargo 2009) and in several numerical studies (e.g., Nolan et al. 2007; Bryan and Rotunno 2009a). It is now well accepted that PI theory offers a reasonable upper bound on balanced axisymmetric TCs in a steady state. On the other hand, several authors have shown that this upper bound may be regulated by a few factors not considered in the theory: turbulent diffusion in the radial direction (Rotunno and Bryan 2012), supergradient wind (Bryan and Rotunno 2009a), intrusion of low-entropy air 
(a) $\mathrm{T}^{\prime}(\mathrm{K})$

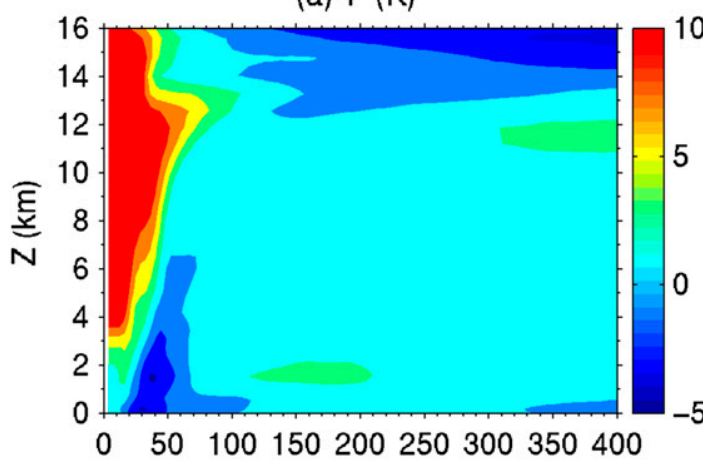

(c) $U \tan (\mathrm{m} / \mathrm{s})$

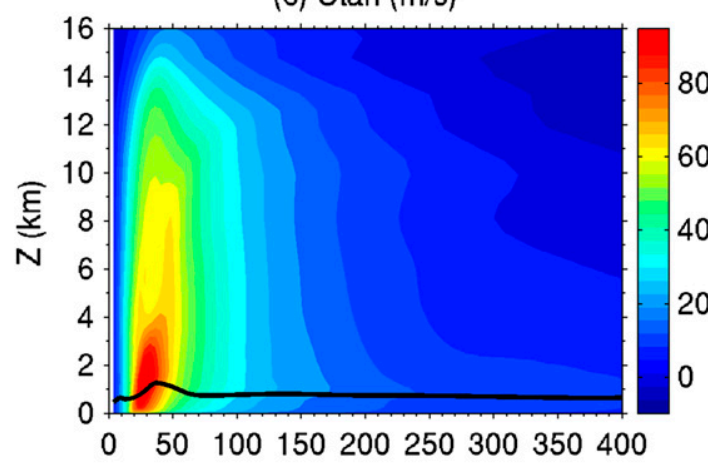

(e) Richardson number

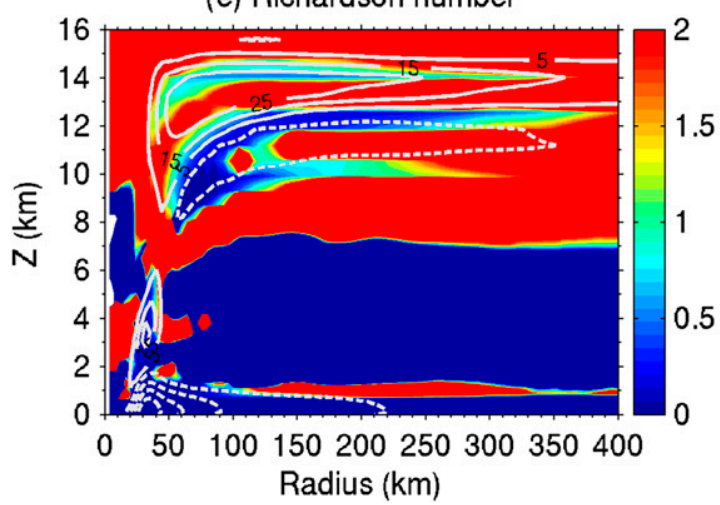

(b) Q' $(g / k g)$

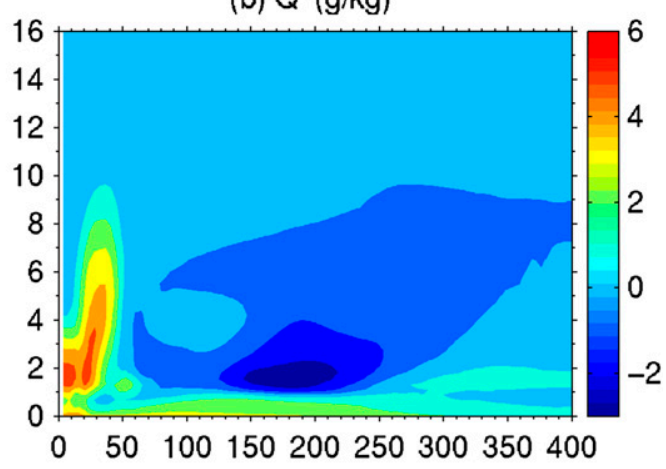

(d) W and Urad (m/s)

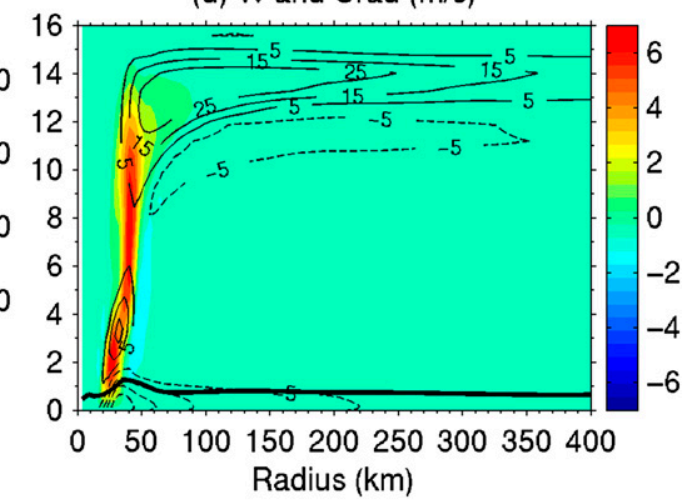

FIG. 4. Axisymmetric structure of the simulated TC with SST $=28^{\circ} \mathrm{C}$ and $T_{o}=205 \mathrm{~K}$. (a) Temperature anomalies with respect to the RCE sounding; (b) moisture anomalies; (c) tangential wind speed (shaded) and boundary layer height (black solid); (d) radial wind (contour interval $=10 \mathrm{~m} \mathrm{~s}^{-1}$; positive: solid; negative: dashed), vertical wind (shaded), and boundary layer height (black solid); (e) $R_{i}$ (shaded) and radial wind (contour interval $=10 \mathrm{~m} \mathrm{~s}^{-1}$ ) with min value reaching $\sim-35 \mathrm{~m} \mathrm{~s}^{-1}$ in the boundary layer. associated with wind shear (Tang and Emanuel 2010, 2012), the highly unbalanced flow in the hurricane boundary layer (Smith et al. 2008; Smith and Montgomery 2010), lateral energy transport (Wang and Xu 2010), and cooling of the upper ocean by TC-induced mixing (Lin et al. 2013). However, a more complete theory relating only environmental fields to TC intensity does not exist yet.

In the axisymmetric framework, values of maximum azimuthal-mean wind speed $V_{\max }$ larger than those predicted by PI theory have been found in several modeling studies (Persing and Montgomery 2003; Hausman et al. 2006; Yang et al. 2007; Bryan and Rotunno 2009b).
Bryan and Rotunno (2009a) attributed this large difference between the PI value and the simulated $V_{\max }$ from an axisymmetric model partly to gradient flow imbalancethe azimuthal flow at eyewall is highly supergradientand partly to the turbulent length scale. Comparisons between PI and the results from 3D models have not been made as systematically as have those with axisymmetric models. In idealized numerical experiments of TCs starting from RCE, Nolan et al. (2007) found that TC intensities reached about $70 \%$ of the PI. We have tested different domain sizes; our results suggest the possibility that the small domain $(1200 \mathrm{~km})$ used by Nolan et al. (2007) may constrain the intensity of the 
TCs. As we increase our domain size, TC intensity increases dramatically even though the environmental soundings are kept the same (see details in the appendix). Consistent with this, Yang et al. (2007) showed in both 2D and 3D numerical experiments maximum wind speeds exceeding PI by more than $20 \mathrm{~m} \mathrm{~s}^{-1}$. Wang and $\mathrm{Xu}$ (2010) found that maximum azimuthally averaged wind from a 3D integration may be about $25 \%-40 \%$ stronger than PI. They suggested that lateral energy transport contributes significantly to local energy balance in the eyewall and that the local energy balance may be of limited use.

Since the PI theory assumes gradient wind balance, we now discuss the gradient wind speed $V_{g}$ and flow imbalance in our 3D numerical experiments. We compute $V_{g}$ as a surrogate for azimuthal gradient wind:

$$
\frac{V_{g}^{2}}{r}+f r=-\frac{1}{\rho} \frac{\partial p}{\partial r}
$$

where $r$ is the radius, $p$ is the pressure, $\rho$ is the density, and $f$ is the Coriolis parameter. Figure 5 shows the radial distribution of both simulated tangential wind and gradient wind $V_{g}$ averaged from days 3.75 to 5 , at the height of the maximum wind speed $(\sim 1.3 \mathrm{~km})$. In contrast to $V_{\max }$, the maximum value of $V_{g}$ is $\sim 85 \mathrm{~m} \mathrm{~s}^{-1}$ and located at approximately $50 \mathrm{~km}$, while $V_{g}$ is $\sim 75 \mathrm{~m} \mathrm{~s}^{-1}$ at the RMW (as defined by the actual wind). This indicates that supergradient effects are largely responsible for the discrepancies between PI and $V_{\max }$, which is close to PI $\left(\sim 80 \mathrm{~m} \mathrm{~s}^{-1}\right.$; see Fig. $2 \mathrm{~b}$, Table 2$)$, if we use the environmental value of $C_{k} / C_{d} \sim 0.9$.

One can further assess the momentum balance in the radial direction. In a quasi-steady state, the equation for the radial momentum in an inviscid flow may be written in cylindrical coordinates as

$$
\overline{u \frac{\partial u}{\partial r}}+\frac{\bar{v} \frac{\partial u}{r}}{\partial \theta}+\overline{w \frac{\partial u}{\partial z}}=\overline{\frac{v^{2}}{r}-\frac{v_{g}^{2}}{r}},
$$

where $u, v$, and $w$ are radial, azimuthal, and vertical winds, respectively, and the overbars denote azimuthal and temporal averages. Each term in Eq. (7) is evaluated using the model output at $1.3 \mathrm{~km}$. Figure $5 \mathrm{~b}$ shows that the right-hand side of Eq. (7), a measure of the degree of departure from gradient wind balance, is $\sim 0.18 \mathrm{~m} \mathrm{~s}^{-2}$ at RMW and is largely balanced by the momentum transport of the vertical advection $\left(\sim 0.15 \mathrm{~m} \mathrm{~s}^{-2}\right)$. The gradient wind imbalance might be incorporated in the PI theory, as discussed in Bryan and Rotunno (2009a), who find that the PI theory with this modification is useful for explaining the difference between PI and the axisymmetric model-simulated maximum azimuthal wind speed. Nevertheless, this appears to be insufficient for

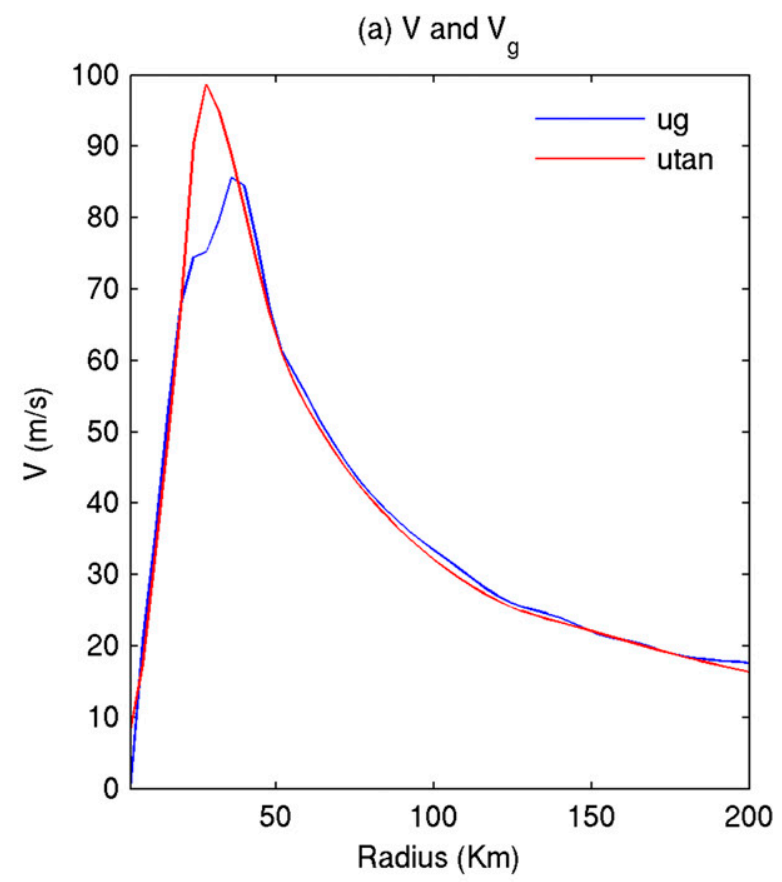

(b) Radial momentum tendency

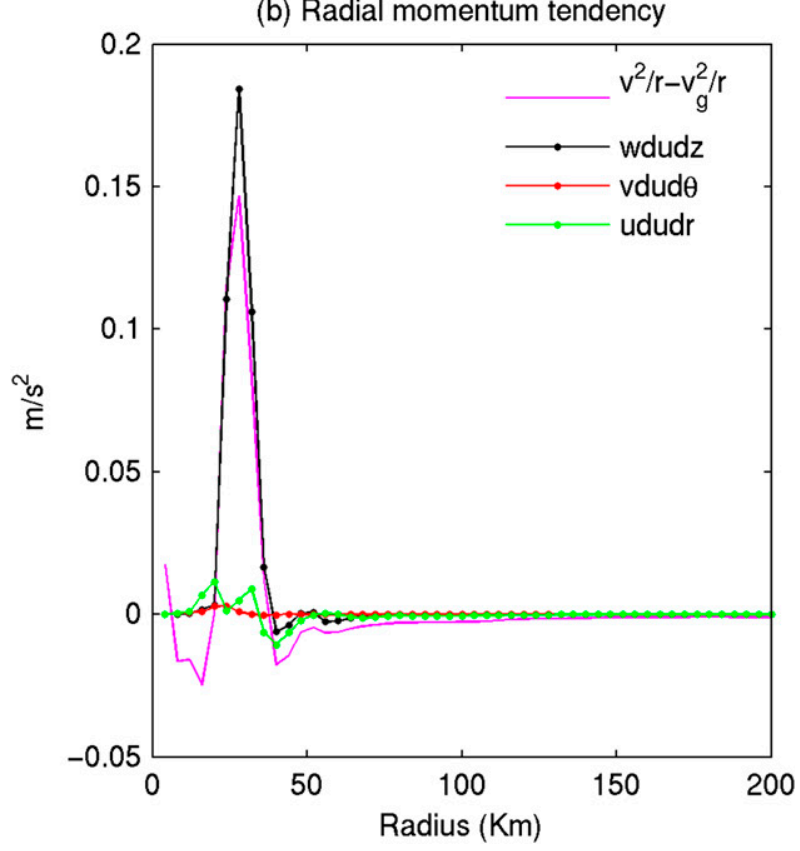

FIG. 5. (a) Azimuthally averaged tangential wind $u_{\text {tan }}$ and tangential gradient wind $u_{g}$. (b) Various terms in Eq. (7) for the radial momentum.

explaining the difference in the gradient wind between the 3D experiments and PI. Possible reasons for this might be that these TCs are not truly in steady state and/ or turbulent momentum mixing is not taken into account in Eq. (7). 
(a) $U \tan (\mathrm{m} / \mathrm{s})$ at $\mathrm{T}_{0}=215 \mathrm{~K}$

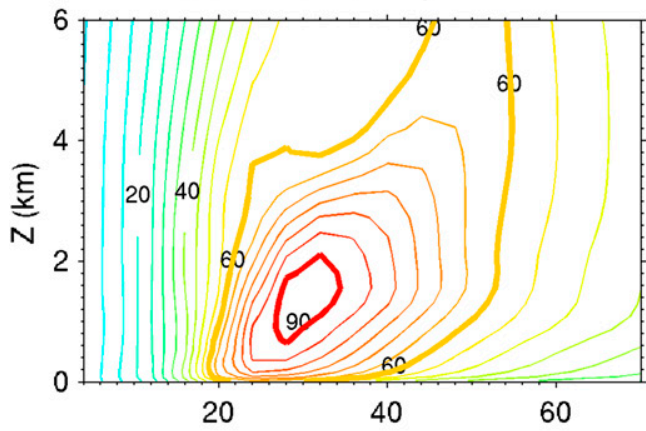

(c) Utan $(\mathrm{m} / \mathrm{s})$ at $\mathrm{T}_{0}=205 \mathrm{~K}$

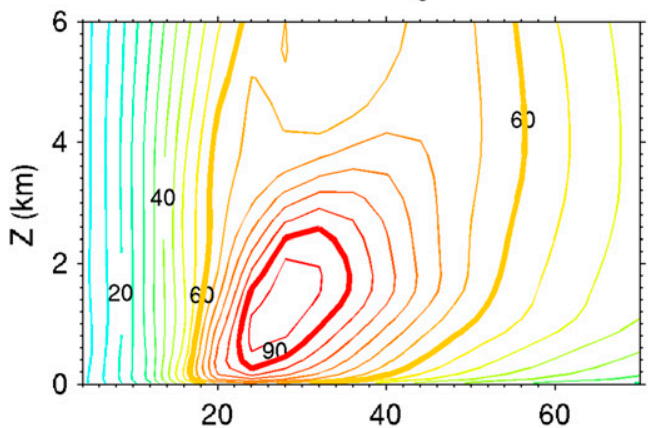

(e) $U \tan (\mathrm{m} / \mathrm{s})$ at $\mathrm{T}_{0}=195 \mathrm{~K}$

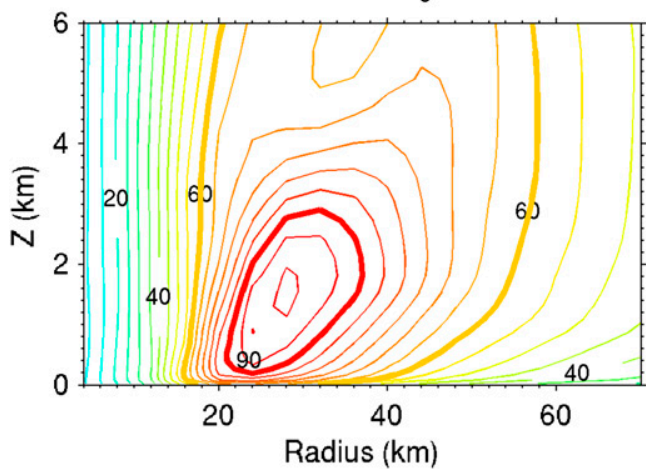

(b) Richardson number at $\mathrm{T}_{\mathrm{o}}=215 \mathrm{~K}$

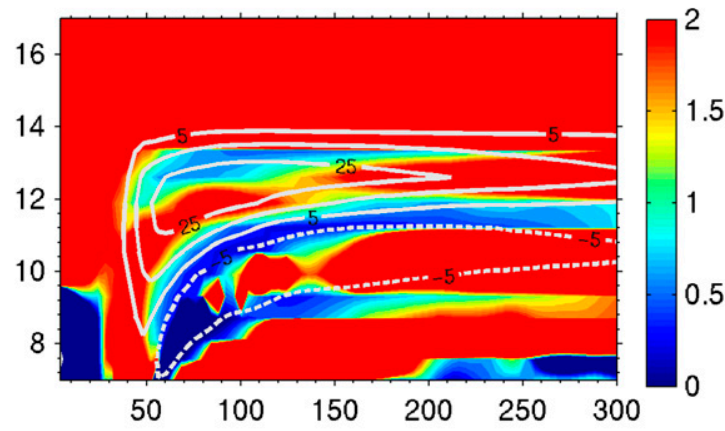

(d) Richardson number at $\mathrm{T}_{0}=205 \mathrm{~K}$

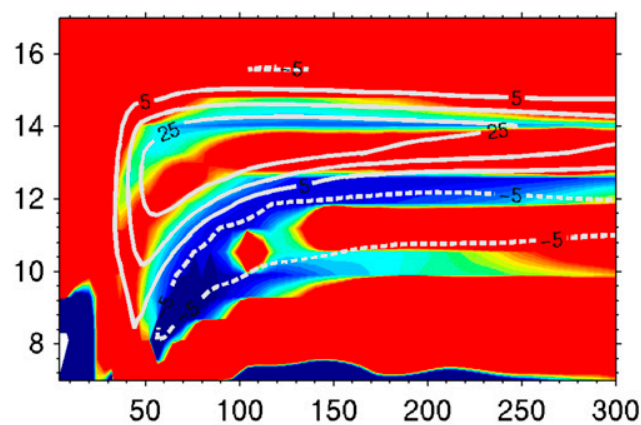

(f) Richardson number at $\mathrm{T}_{0}=195 \mathrm{~K}$

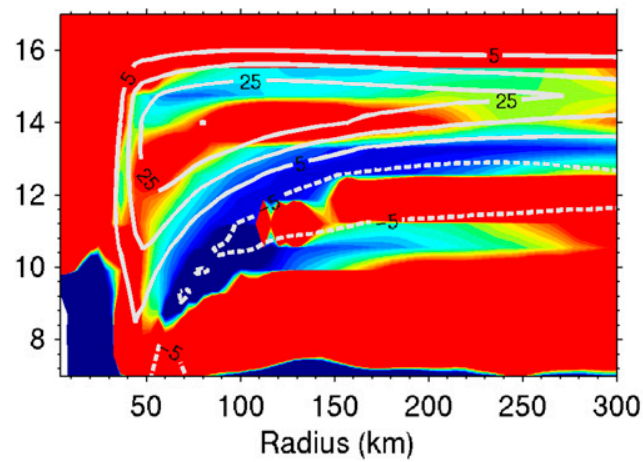

FIG. 6. (left) Tangential wind (contour interval $=5 \mathrm{~m} \mathrm{~s}^{-1} ; 60$ and $90 \mathrm{~m} \mathrm{~s}^{-1}$ are thick) and (right) radial wind (contour interval $=5 \mathrm{~m} \mathrm{~s}^{-1}$ with the zero contour suppressed) with $R_{i}$ (shaded) averaged from days 3.75 to 5 for three $3 \mathrm{D}$ experiments at SST $=28^{\circ} \mathrm{C}$ : (a),(b) $T_{o}=215$; (c),(d) $T_{o}=205$; and (e),(f) $T_{o}=195 \mathrm{~K}$.

\section{3) TC INTENSITY AS A FUNCTION OF $T_{O}$}

We now turn to the impact of $T_{o}$ on the intensity of the TCs. Figure 6 shows the low-level maximum tangential wind speed, upper-level radial wind, and Richardson number averaged from days 3.75 to 5 for three experiments: $T_{o}=195,205$, and $215 \mathrm{~K}$ at SST $=28^{\circ} \mathrm{C}$. These plots show that the colder tropopause and stratosphere does not alter the TC structure in these experiments. TC intensity measured as the maximum tangential wind speed increases from less than $95 \mathrm{~m} \mathrm{~s}^{-1}$ with $T_{o}=215 \mathrm{~K}$ to more than $100 \mathrm{~m} \mathrm{~s}^{-1}$ with $T_{o}=195 \mathrm{~K}$, and area coverage by the
$90 \mathrm{~m} \mathrm{~s}^{-1}$ contour in the radial-height plane increases substantially. As $T_{o}$ is decreased from 215 to $195 \mathrm{~K}$, the outflow jet shifts upward, since the tropopause rises in the RCE soundings (Fig. 1); the outflow jets strengthen slightly and their spatial structure remains the same.

Figure 7 shows the time series of the minimum sea level pressure and the maximum azimuthal-mean wind for the experiments with different values of $T_{o}$, from 195 to $215 \mathrm{~K}$. The minimum sea level pressure is lowest around day 4 and persists for about 1-1.5 days, in the range $885-910 \mathrm{hPa}$ for all the experiments during this period, and increases afterward as the TCs weaken. The 
(a) Minimum sea level pressure $(\mathrm{hPa})$

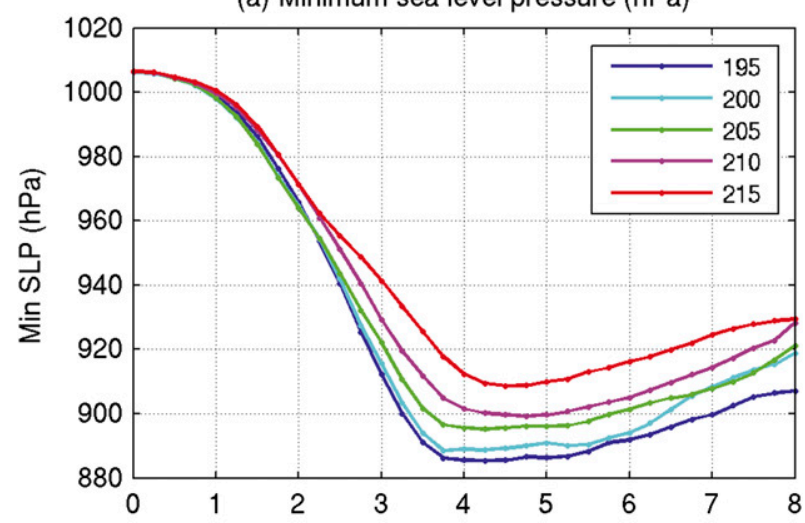

(b) Maximum tangential wind $(\mathrm{m} / \mathrm{s})$

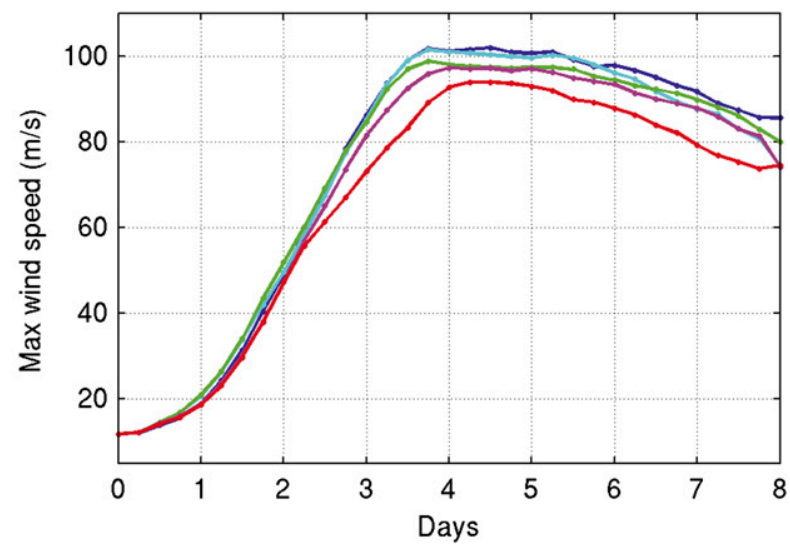

FIG. 7. Time series of (a) min sea level pressure (hPa; every $6 \mathrm{~h}$ ) and (b) max tangential wind speed $\left(\mathrm{m} \mathrm{s}^{-1}\right.$; every $\left.6 \mathrm{~h}\right)$ at SST $=28^{\circ} \mathrm{C}$ for five different values of $T_{o}(195,200,205,210$, and $215 \mathrm{~K})$. A three-point smoothing is applied for all the time series.

intensities of these transient TC solutions, as measured by minimum sea level pressure, increase with decreasing tropopause temperature, by $\sim 1 \mathrm{hPa}$ per $1 \mathrm{~K}$ of cooling.

The time series of the maximum azimuthal wind speed (Fig. 7b) show maximum values from 95 to $102 \mathrm{~m} \mathrm{~s}^{-1}$, reached between days 3.75 and 5. Unlike the minimum sea level pressure, the azimuthal wind is subject to aliasing error in the conversion from Cartesian to cylindrical coordinates. Figure 8 shows the maximum azimuthal wind versus $T_{o}$ for all the experiments with 4-km horizontal grid spacing over three different values of SST: $26^{\circ}, 28^{\circ}$, and $30^{\circ} \mathrm{C}$. The impact of the tropopause temperature on the TC at each SST is evident. Taking all these experiments together, linear regression yields a $0.46 \mathrm{~m} \mathrm{~s}^{-1}$ increase of maximum azimuthal wind for every degree cooling at the tropopause. The $95 \%$ confidence interval of the estimated slope from the Student's $t$ test is from -0.72 to -0.20 (computed using the MATLAB function "fit"). The maximum gradient wind, as discussed

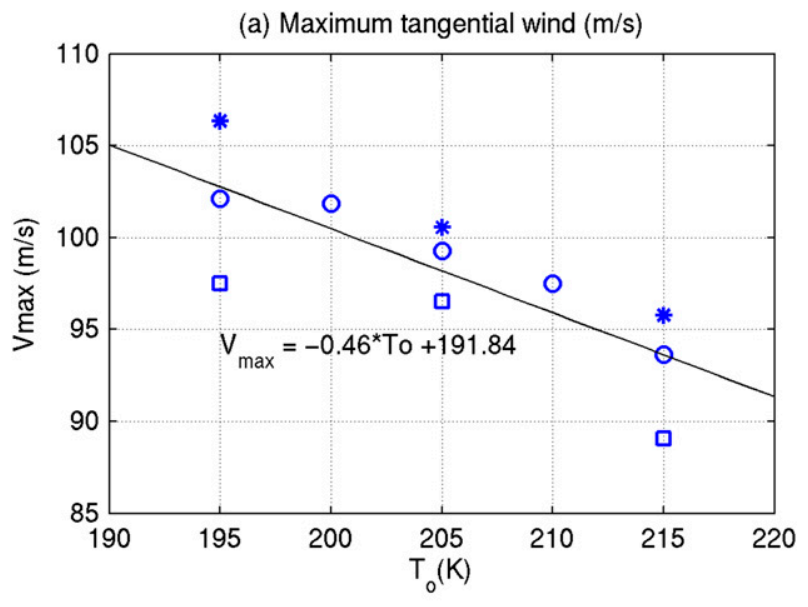

(b) Maximum tangential gradient wind $(\mathrm{m} / \mathrm{s})$

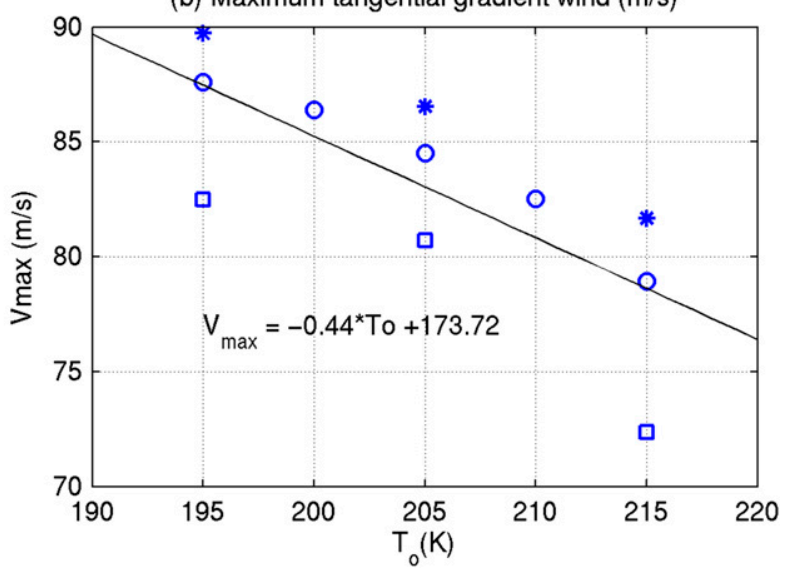

FIG. 8. Time-averaged (a) max tangential wind and (b) max gradient wind using 6-hourly model output from days 3.75 to 5 . Squares denote SST $=26^{\circ} \mathrm{C}$, circles denote SST $=28^{\circ} \mathrm{C}$, and asterisks denote SST $=30^{\circ} \mathrm{C}$. The $95 \%$ confidence interval of the estimated slope is from -0.72 to -0.20 for the max tangential wind in (a) and from -0.74 to -0.14 for the max gradient wind in (b).

previously, is weaker, $\sim 70 \%$ of the maximum azimuthal wind in all the experiments. Figure $8 b$ shows that the peak gradient wind from each experiment scales similarly to the actual wind with $T_{o}$.

\section{4) SEnSitivity to RESOLUtion}

In this section, we examine the sensitivity of our TC solutions, including TC intensity and its dependence on the tropopause temperature, to horizontal resolution. In this set of experiments, we use $8-\mathrm{km}$ horizontal grid spacing in the inner domain and $24 \mathrm{~km}$ in the outer domain, while keeping the same domain size and physical parameterization schemes. The environmental soundings are generated from RCE integrations at $D x=8 \mathrm{~km}$, which yields similar PI- $T_{o}$ slope, $\sim-0.4 \mathrm{~m} \mathrm{~s}^{-1} \mathrm{~K}^{-1}$ (black squares in Fig. 2). 
As the grid spacing increases from 4 to $8 \mathrm{~km}$, TC intensity decreases, as expected, because the frontal structure at RMW is more diffuse at lower resolution. For example, the maximum wind speed at $T_{o}=200 \mathrm{~K}$ decreases from $105 \mathrm{~m} \mathrm{~s}^{-1}$ with $D x=4 \mathrm{~km}$ to $90 \mathrm{~m} \mathrm{~s}^{-1}$ with $D x=8 \mathrm{~km}$ and $\mathrm{SST}=28^{\circ} \mathrm{C}$. Figure 9 shows simulated maximum azimuthally averaged wind speed as a function of $T_{o}$. The slope between hurricane intensity and $T_{o}$ increases slightly to $\sim-0.5 \mathrm{~m} \mathrm{~s}^{-1} \mathrm{~K}^{-1}$ with the $95 \%$ confidence interval from -0.59 to -0.4 .

\section{TCs in statistical equilibrium}

In this section, we examine TC intensity in equilibrium states. We perform five convective-permitting experiments with the same range of values for the tropopause temperature $T_{o}: 195,200,205,210$, and $215 \mathrm{~K}$. To reduce computation cost, we use a smaller domain size $(3200 \mathrm{~km})$ with doubly periodic lateral boundary conditions and $D x=8 \mathrm{~km}$, but we keep the same physics and the number of vertical levels as in the previous experiments. Using lower horizontal resolution of $8 \mathrm{~km}$ may be justified by the fact that the model still produces a similar relation between the tropopause temperature and TC intensity as that obtained with $D x=4 \mathrm{~km}$, although the simulated TC intensities overall are reduced. We do not use the nesting technique in these long integrations. The model is integrated for 200 days to obtain adequate statistics of TC intensity in the equilibrium state. In RCE, some aspects of the model solutions are well constrained; for example, the time- and domainaveraged rainfall equals the evaporation, as required by the equilibrium water vapor budget, and the vertically integrated radiative cooling is in balance with the net surface enthalpy flux because of the conservation of moist static energy. In these experiments, both the domainaveraged evaporation and rainfall are $\sim 4.1 \mathrm{~mm} \mathrm{day}^{-1}$ and vary by about $10 \%$ in its time mean over the parameter range of $T_{o}$ in these RCE states.

Figure 10 displays the time series of both the minimum sea level pressure and maximum azimuthal-mean wind speed. During the first 10 days, the maximum azimuthal wind speed varies between 80 and $90 \mathrm{~m} \mathrm{~s}^{-1}$. The peak intensities of these TCs also scale similarly with $T_{o}$, consistent with previous results. After this first peak, the TCs weaken substantially but maintain coherent axisymmetric structures. It is also evident in Fig. 10 (top) that a colder tropopause not only yields larger intensities (e.g., in the first 10 days), but also larger temporal oscillations throughout the first 100 days. The strengthening-weakening cycle repeats a few times before a statistical equilibrium is established. (a) Minimum sea level pressure $(\mathrm{hPa})$

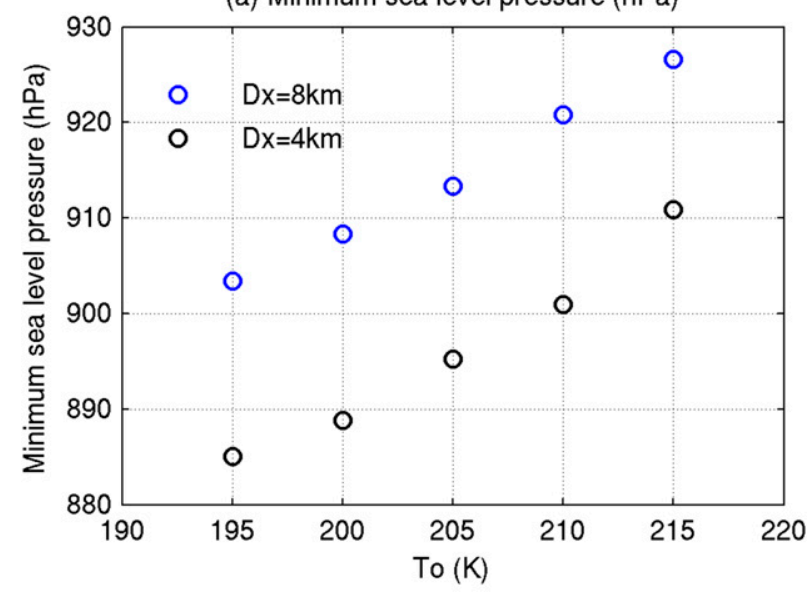

(b) Maximum tangential wind $(\mathrm{m} / \mathrm{s})$

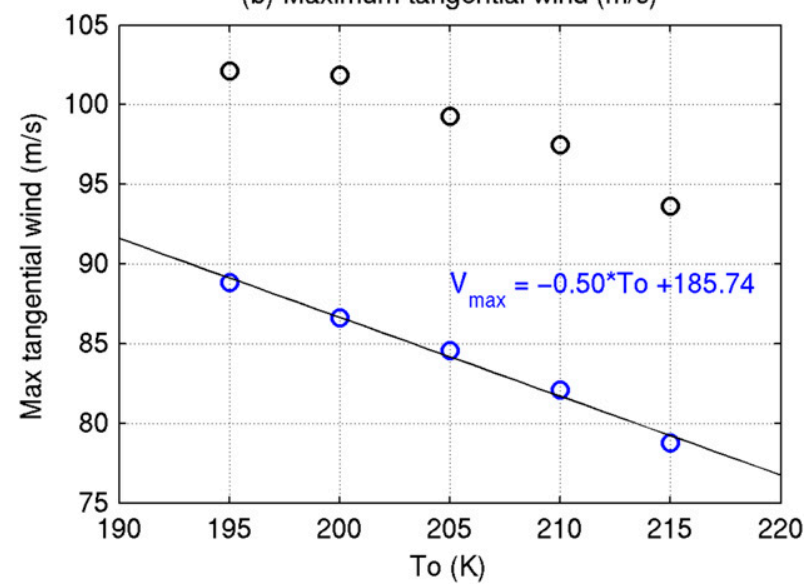

FIG. 9. (a) Min sea level pressure and (b) max tangential wind speed from the experiments at $\mathrm{SST}=28^{\circ} \mathrm{C}$, but with horizontal grid spacing $D x=8 \mathrm{~km}$ (blue circles). Results from $D x=4 \mathrm{~km}$ (black circles) are also shown for reference. The $95 \%$ confidence interval of the estimated slope in (b) is from -0.59 to -0.4 .

During this transition stage, the free-tropospheric temperature warms by several kelvins (not shown) in the TC RCE state over that in the pure RCE state, indicating that the environment has been substantially modified by the presence of TCs. After approximately 100 days (similar to the time scale to reach the non-TC RCE), the model solution eventually settles into a statistical equilibrium. In this equilibrium the TC intensities, measured in either pressure or winds, oscillate irregularly. Similar oscillatory behavior of statistically steady TC solutions has been noted in other axisymmetric modeling studies (e.g., Hakim 2011).

Because of the temporal oscillations, it is not immediately clear if the intensities of these TCs scale with the tropopause temperature similarly to those in the transient solutions in the first 10 days. Here, we use two metrics to quantify TC intensity: the time mean and the 

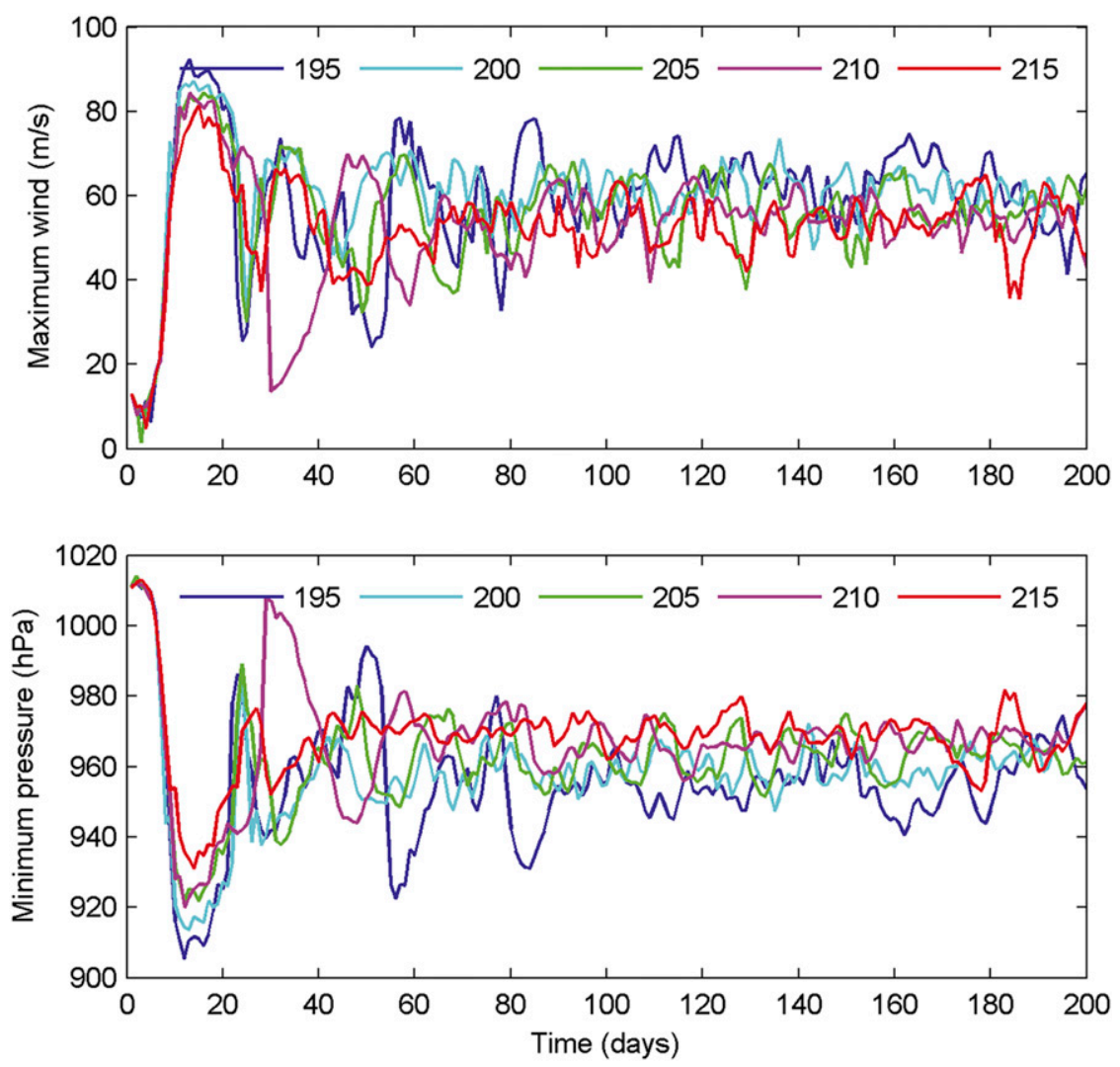

FIG. 10. Time series of the (top) max azimuthal-mean winds and (bottom) min sea level pressure from five 200-day integrations with $T_{o}=195,200,205,210$, and $215 \mathrm{~K}$.

99th percentile of maximum wind speed. Both are computed from the last 100 days of the integrations, after the initial large oscillations have settled down.

The relationship between equilibrium TC intensity and tropopause temperature is shown in Fig. 11 for these five equilibrium experiments. Both the mean intensity and the 99th percentile show a strong relationship to the tropopause temperature. The standard deviation of the mean intensity generally increases with a colder tropopause, although the minimum occurs at $T_{o}=$ $200 \mathrm{~K}$. The coefficient obtained from linear regression is $\sim-0.48 \mathrm{~m} \mathrm{~s}^{-1} \mathrm{~K}^{-1}$ for the mean intensity and $\sim-0.67 \mathrm{~m} \mathrm{~s}^{-1} \mathrm{~K}^{-1}$ for the 99th percentile. This is slightly larger than the values found in the transient TC solutions.

\section{Conclusions}

We have investigated the influence of the tropopause temperature on the intensity of idealized TCs using a 3D mesoscale model in a radiative-convective environment. While the model has "full physics" in all other respects, the radiative cooling is specified to a constant $1.2 \mathrm{~K} \mathrm{day}^{-1}$ in the troposphere, as well as a relaxation to constant tropopause temperature in an isothermal stratosphere.
That imposed tropopause and stratospheric temperature is used as our control parameter and varied over a range of $20 \mathrm{~K}$ in the $3 \mathrm{D}$ numerical experiments.

The potential intensity, calculated using the thermodynamic profiles that are simulated in the small domain in the radiative-convective equilibrium before the TCs

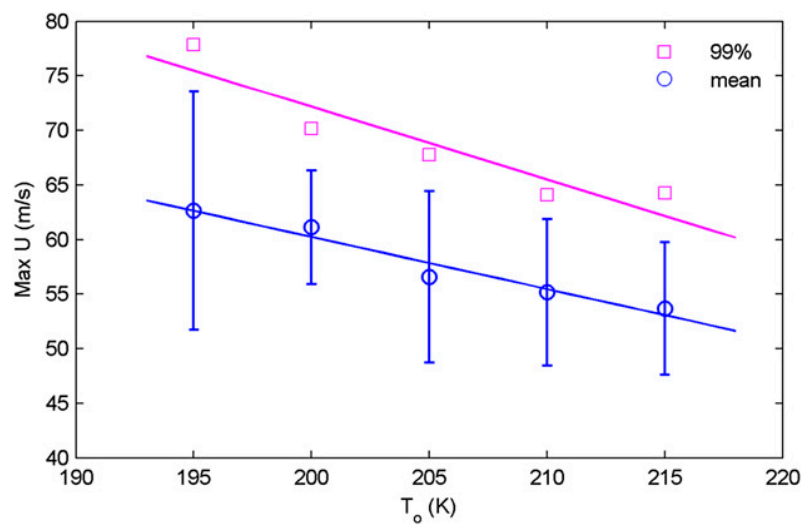

FIG. 11. Max azimuthal-mean wind from the last 100 days of the five equilibrium experiments (Fig. 10) vs $T_{o}$. The linear regression coefficient between $V_{\max }$ and $T_{o}$ is $\sim-0.48 \mathrm{~m} \mathrm{~s}^{-1} \mathrm{~K}^{-1}$ for the mean intensity (blue line) and $\sim-0.67 \mathrm{~m} \mathrm{~s}^{-1} \mathrm{~K}^{-1}$ for the 99th percentile of the max wind speed (magenta line). 
are introduced, yields an increase of $0.4-1 \mathrm{~m} \mathrm{~s}^{-1}$ for each degree cooling at the tropopause. The specific value within this range depends on choices made for several parameters in the PI code, with the ratio of exchange coefficients being the most important of those. The 3D integrations using the WRF Model with horizontal grid spacing of $4 \mathrm{~km}$ yield intense tropical cyclones with maximum azimuthal wind speed exceeding PI by substantial fractions; the magnitude of these excesses are also dependent on the parameters chosen in the PI calculation. Some of this discrepancy is explained by the supergradient wind. On the other hand, the intensity of these 3D TCs show a $\sim 0.4 \mathrm{~m} \mathrm{~s}^{-1}$ increase per degree of temperature decrease at the tropopause in the radiative-convective equilibrium. This value is near the lower end of the PIestimated TC intensity dependence. Sensitivity experiments with horizontal grid spacing of $8 \mathrm{~km}$ produce less intense TCs, as expected, but a similar slope $\left(\sim-0.5 \mathrm{~m} \mathrm{~s}^{-1} \mathrm{~K}^{-1}\right)$ with respect to the tropopause temperature.

Equilibrium TC solutions are obtained in five 200-day experiments, over a range of tropopause temperatures $20 \mathrm{~K}$ wide, with horizontal grid spacing of $8 \mathrm{~km}$ in a $3200-\mathrm{km}$-wide numerical domain. These equilibrium TC solutions have maximum wind speeds in the range of $40-60 \mathrm{~m} \mathrm{~s}^{-1}$ and minimum sea level pressures in the range of $940-970 \mathrm{hPa}$, and they exhibit significant temporal viability. When the TC intensity in these solutions is measured using either the time mean or the 99th percentile of maximum wind speed, both quantities show a strong relationship with the tropopause temperature. The linear regression coefficient is $\sim-0.48 \mathrm{~m} \mathrm{~s}^{-1} \mathrm{~K}^{-1}$ for the mean intensity, and $\sim-0.67 \mathrm{~m} \mathrm{~s}^{-1} \mathrm{~K}^{-1}$ for the 99th percentile of the maximum wind speed.

Finally, we note that the dependence of these transient and steady-state 3D TC solutions on the tropopause temperature differs quantitatively from that found by Ramsay (2013, his Fig. 3), who performed idealized axisymmetric TC experiments and showed $\sim 1 \mathrm{~m} \mathrm{~s}^{-1}$ increase with $1-\mathrm{K}$ cooling in the tropopause temperature. There are other differences, aside from the difference in the dimension, that may be responsible for quantitative discrepancy; for example, Ramsay (2013) used a fixed ratio of $C_{k}$ and $C_{d}(0.5)$ and dissipative heating, while $C_{k} / C_{d}$ varies greatly in $3 \mathrm{D}$ simulations and the effect of dissipative heating is too weak in our 3D TC experiments. Although the discrepancy for the different behavior in 2D TC experiments of Ramsay (2013) and our 3D TC experiments study remains to be resolved, both studies have demonstrated that tropopause temperature has a detectable impact on the TC intensity.

Acknowledgments. The early phases of this work, particularly the simulation design and initial numerical integrations, were supported by NOAA Grants

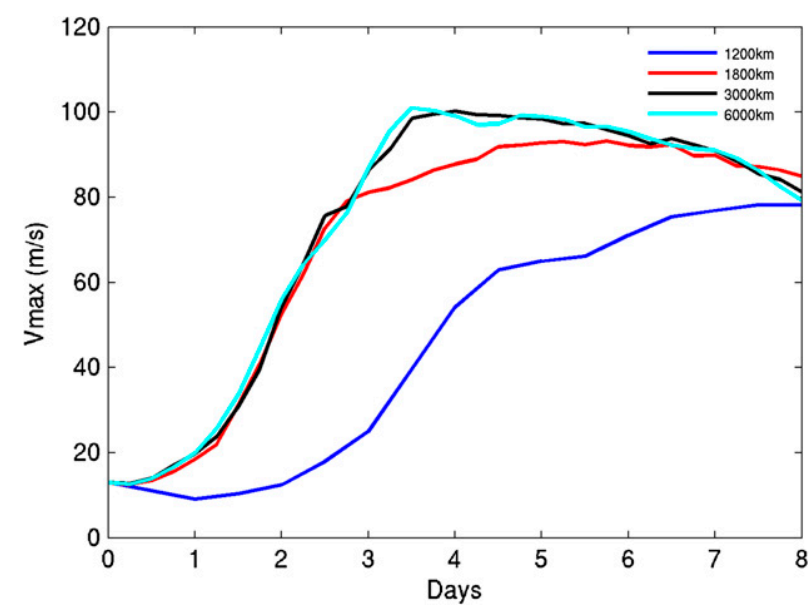

FIG A1. Time series of the max azimuthal-mean tangential wind speed from four experiments with different domain size: 1200, 1800,3000 , and $6000 \mathrm{~km}$. Note that in the $1200-\mathrm{km}$ integration, no nesting is used.

NA08OAR4320912 and NA11OAR4310093. The later stages, including analysis and manuscript preparation, were supported by NSF Grant AGS-1008847. L.M.P. is supported, in part, by NSF Grant AGS-1322439. We are thankful to Prof. Kerry Emanuel for sharing his PI code and for his suggestion of Eqs. (4) and (5). We thank NCAR's Computational Information Systems Laboratory, where part of the model integrations was performed. We also thank Dr. Gustavo J. P. Correa for maintaining the Linux cluster where some of model integrations were performed. We are grateful for Dr. Robert Korty and two anonymous reviewers for their insightful comments.

\section{APPENDIX}

\section{Domain Size Dependence of Hurricane Intensity}

The size of the outer computational domain is $6000 \mathrm{~km}$ in the 3D nesting experiments, and $3200 \mathrm{~km}$ in the statistical equilibrium integrations. Domain size can influence TC intensity, as found previously in axisymmetric models (Chavas and Emanuel 2014). We have experimented different domain size for the same parameters SST $=28^{\circ} \mathrm{C}$ and $T_{o}=205 \mathrm{~K}$. We use three different size of the outer domain $(1800,3000$, and $6000 \mathrm{~km})$ in the 3D experiments, while keeping the inner domain the same. In one additional experiment, only the inner domain $(1200 \mathrm{~km})$ is used. Fig. A1 shows that the TC peak intensity in the 3000and 6000-km experiments is nearly the same $\left(\sim 100 \mathrm{~m} \mathrm{~s}^{-1}\right)$, except that the latter shows more temporal viability. The maximum wind speed is reduced to $90 \mathrm{~m} \mathrm{~s}^{-1}$ in the $1800 \mathrm{~km}$ experiment, and $80 \mathrm{~m} \mathrm{~s}^{-1}$ in the $1200 \mathrm{~km}$ experiment. The timing of peak intensity is also delayed in smaller domains. 


\section{REFERENCES}

Bell, M. M., M. T. Montgomery, and K. A. Emanuel, 2012: Air-sea enthalpy and momentum exchange at major hurricane wind speeds observed during CBLAST. J. Atmos. Sci., 69, 31973222, doi:10.1175/JAS-D-11-0276.1.

Bister, M., and K. A. Emanuel, 1997: The genesis of Hurricane Guillermo: TEXMEX analyses and a modeling study. Mon. Wea. Rev., 125, 2662-2682, doi:10.1175/1520-0493(1997)125<2662: TGOHGT $>2.0 . \mathrm{CO} ; 2$.

—_ and _ 1998: Dissipative heating and hurricane intensity. Meteor. Atmos. Phys., 65, 233-240, doi:10.1007/BF01030791. , and _- 2002: Low frequency variability of tropical cyclone potential intensity: 1 . Interannual to interdecadal variability. J. Geophys. Res., 107, 4801, doi:10.1029/2001JD000776.

Black, P. G., and Coauthors, 2007: Air-sea exchange in hurricanes: Synthesis of observations from the Coupled Boundary Layer Air-Sea Transfer experiment. Bull. Amer. Meteor. Soc., 88, 357-374, doi:10.1175/BAMS-88-3-357.

Bryan, G. H., and R. Rotunno, 2009a: Evaluation of an analytical model for the maximum intensity of tropical cyclones. $J$. Atmos. Sci., 66, 3042-3060, doi:10.1175/2009JAS3038.1.

— and $-2009 \mathrm{~b}$ : The maximum intensity of tropical cyclones in axisymmetric numerical model simulations. Mon. Wea. Rev., 137, 1770-1789, doi:10.1175/2008MWR2709.1.

Chavas, D. R., and K. A. Emanuel, 2014: Equilibrium tropical cyclone size in an idealized state of axisymmetric radiativeconvective equilibrium. J. Atmos. Sci., 71, 1663-1680, doi:10.1175/ JAS-D-13-0155.1.

Chen, S.-H., and W.-Y. Sun, 2002: A one-dimensional time dependent cloud model. J. Meteor. Soc. Japan, 80, 99-118, doi:10.2151/jmsj.80.99.

Craig, G. C., and S. L. Gray, 1996: CISK or WISHE as the mechanism for tropical cyclone intensification. J. Atmos. Sci., 53, 3528-3540, doi:10.1175/1520-0469(1996)053<3528:COWATM>2.0.CO;2.

Emanuel, K. A., 1987: An air-sea interaction model of intraseasonal oscillations in the tropics. J. Atmos. Sci., 44, 2324-2340, doi:10.1175/1520-0469(1987)044<2324:AASIMO>2.0.CO;2.

_ 2000: A statistical analysis of tropical cyclone intensity. Mon. Wea. Rev., 128, 1139-1152, doi:10.1175/1520-0493(2000)128<1139: ASAOTC $>2.0 . \mathrm{CO} ; 2$

Emanuel, K., 2010: Tropical cyclone activity downscaled from NOAA-CIRES Reanalysis, 1908-1958. J. Adv. Model. Earth Syst., 2 (1), doi:10.3894/JAMES.2010.2.1.

_ 2012 : Self-stratification of tropical cyclone outflow. Part II: Implications for storm intensification. J. Atmos. Sci., 69, 988 996, doi:10.1175/JAS-D-11-0177.1.

_ and R. Rotunno, 2011: Self-stratification of tropical cyclone outflow. Part I: Implications for storm structure. J. Atmos. Sci., 68, 2236-2249, doi:10.1175/JAS-D-10-05024.1.

- S. Solomon, D. Folini, S. Davis, and C. Cagnazzo, 2013: Influence of tropical tropopause layer cooling on Atlantic hurricane activity. J. Climate, 26, 2288-2301, doi:10.1175/JCLI-D-12-00242.1.

Hakim, G. J., 2011: The mean state of axisymmetric hurricanes in statistical equilibrium. J. Atmos. Sci., 68, 1364-1376, doi:10.1175/ 2010JAS3644.1.

Haus, B. K., D. Jeong, M. A. Donelan, J. A. Zhang, and I. Savelyev, 2010: Relative rates of sea-air heat transfer and frictional drag in very high winds. Geophys. Res. Lett., 37, L07802, doi:10.1029/ 2009GL042206.

Hausman, S. A., K. V. Ooyama, and W. H. Schubert, 2006: Potential vorticity structure of simulated hurricanes. J. Atmos. Sci., 63, 87-108, doi:10.1175/JAS3601.1.
Hill, K. A., and G. M. Lackmann, 2011: The impact of future climate change on TC intensity and structure: A downscaling approach. J. Climate, 24, 4644-4661, doi:10.1175/ 2011JCLI3761.1.

Hong, S.-Y., Y. Noh, and J. Dudhia, 2006: A new vertical diffusion package with an explicit treatment of entrainment processes. Mon. Wea. Rev., 134, 2318-2341, doi:10.1175/MWR3199.1.

Kalnay, E., and Coauthors, 1996: The NCEP/NCAR 40-Year Reanalysis Project. Bull. Amer. Meteor. Soc., 77, 437-471, doi:10.1175/1520-0477(1996)077,0437:TNYRP.2.0.CO;2.

Kepert, J. D., 2001: The dynamics of boundary layer jets within the tropical cyclone core. Part I: Linear theory. J. Atmos. Sci., 58, 2469-2484, doi:10.1175/1520-0469(2001)058<2469: TDOBLJ $>2.0 . \mathrm{CO} ; 2$.

- 2012: Choosing a boundary layer parameterization for tropical cyclone modeling. Mon. Wea. Rev., 140, 1427-1445, doi:10.1175/MWR-D-11-00217.1.

Klemp, J. B., J. Dudhia, and A. D. Hassiotis, 2008: An upper gravity-wave absorbing layer for NWP applications. Mon. Wea. Rev., 136, 3987-4004, doi:10.1175/2008MWR2596.1.

Knutson, T. R., R. E. Tuleya, J. J. Sirutis, G. A. Vecchi, and I. Held, 2008: Simulated reduction in Atlantic hurricane frequency under twenty-first- century warming conditions. Nat. Geosci., 1, 359-364, doi:10.1038/ngeo202.

Kossin, J. P., and S. J. Camargo, 2009: Hurricane track variability and secular potential intensity trends. Climatic Change, 97, 329-337, doi:10.1007/s10584-009-9748-2.

Lin, I.-I., and Coauthors, 2013: An ocean coupling potential intensity index for tropical cyclones. Geophys. Res. Lett., 40, 1878-1882, doi:10.1002/grl.50091.

Lin, Y.-L., R. D. Farley, and H. D. Orville, 1983: Bulk parameterization of the snow field in a cloud model. J. Climate Appl. Meteor., 22, 1065-1092, doi:10.1175/1520-0450(1983)022<1065: BPOTSF $>2.0 . \mathrm{CO} ; 2$.

Molinari, J., P. Duran, and D. Vollaro, 2014: Low Richardson number in the tropical cyclone outflow layer.J. Atmos. Sci., 71, 3164-3179, doi:10.1175/JAS-D-14-0005.1.

Montgomery, M. T., M. E. Nicholls, T. A. Cram, and A. B. Saunders, 2006: A vortical hot tower route to tropical cyclogenesis. J. Atmos. Sci., 63, 355-386, doi:10.1175/JAS3604.1.

Mrowiec, A., S. T. Garner, and O. M. Pauluis, 2011: Axisymmetric hurricane in a dry atmosphere: Theoretical framework and numerical experiments. J. Atmos. Sci., 68, 1607-1619, doi:10.1175/2011JAS3639.1.

Nolan, D. S., E. D. Rappin, and K. A. Emanuel, 2007: Tropical cyclogenesis sensitivity to environmental parameters in radiativeconvective equilibrium. Quart. J. Roy. Meteor. Soc., 133, 20852107, doi:10.1002/qj.170.

— J. A. Zhang, and D. P. Stern, 2009a: Evaluation of planetary boundary layer parameterizations in tropical cyclones by comparison of in situ observations and high-resolution simulations of Hurricane Isabel (2003). Part I: Initialization, maximum winds, and the outer-core boundary layer. Mon. Wea. Rev., 137, 3651-3674, doi:10.1175/2009MWR2785.1.

D. P. Stern, and J. A. Zhang, 2009b: Evaluation of planetary boundary layer parameterizations in tropical cyclones by comparison of in situ observations and high-resolution simulations of Hurricane Isabel (2003). Part II: Inner-core boundary layer and eyewall structure. Mon. Wea. Rev., 137, 3675-3698, doi:10.1175/2009MWR2786.1.

Ooyama, K., 1969: Numerical simulation of the life cycle of tropical cyclones. J. Atmos. Sci., 26, 3-40, doi:10.1175/ 1520-0469(1969)026<0003:NSOTLC $>2.0 . \mathrm{CO} ; 2$ 
Pauluis, O., and S. Garner, 2006: Sensitivity of radiative-convective equilibrium simulations to horizontal resolution. J. Atmos. Sci., 63, 1910-1923, doi:10.1175/JAS3705.1.

Persing, J., and M. T. Montgomery, 2003: Hurricane superintensity. J. Atmos. Sci., 60, 2349-2371, doi:10.1175/1520-0469(2003)060<2349: $\mathrm{HS}>2.0 . \mathrm{CO} ; 2$.

,-- J J. C. McWilliams, and R. K. Smith, 2013: Asymmetric and axisymmetric dynamics of tropical cyclones. Atmos. Chem. Phys., 13, 12 299-12 341, doi:10.5194/acp-13-12299-2013.

Ramsay, H. A., 2013: The effects of imposed stratospheric cooling on the maximum intensity of tropical cyclones in axisymmetric radiative-convective equilibrium. J. Climate, 26, 9977-9985, doi:10.1175/JCLI-D-13-00195.1.

, and A. H. Sobel, 2011: Effects of relative and absolute sea surface temperature on tropical cyclone potential intensity using a single-column model. J. Climate, 24, 183-193, doi:10.1175/ 2010JCLI3690.1.

Randel, W. J., and Coauthors, 2009: An update of observed stratospheric temperature trends. J. Geophys. Res., 114, D02107, doi:10.1029/2008JD010421.

Rappin, E. D., D. S. Nolan, and K. A. Emanuel, 2010: Thermodynamic control of tropical cyclogenesis in environments of radiative-convective equilibrium with shear. Quart. J. Roy. Meteor. Soc., 136, 1954-1971, doi:10.1002/qj.706.

Raymond, D. J., S. Sessions, and Z. Fuchs, 2007: A theory for the spinup of tropical depressions. Quart. J. Roy. Meteor. Soc., 133, 1743-1754, doi:10.1002/qj.125.

Rotunno, R., and K. A. Emanuel, 1987: An air-sea interaction theory for tropical cyclones, Part II: Evolutionary study using axisymmetric nonhydrostatic numerical model. J. Atmos. Sci., 44, 542-561, doi:10.1175/1520-0469(1987)044<0542: AAITFT $>2.0 . \mathrm{CO} ; 2$.

— on simulated hurricanes. J. Atmos. Sci., 69, 2284-2299, doi:10.1175/JAS-D-11-0204.1.

Skamarock, W. C., and Coauthors, 2008: A description of the Advanced Research WRF version 3. NCAR Tech. Note NCAR/TN-475+STR, 113 pp., doi:10.5065/D68S4MVH.

Smith, R. K., and S. Vogl, 2008: A simple model of the hurricane boundary layer revisited. Quart. J. Roy. Meteor. Soc., 134, 337351, doi:10.1002/qj.216.

— theory. Quart. J. Roy. Meteor. Soc., 136, 1665-1670, doi:10.1002/ qj.679.

- — , and S. Vogl, 2008: A critique of Emanuel's hurricane model and potential intensity theory. Quart. J. Roy. Meteor. Soc., 134, 551-561, doi:10.1002/qj.241.
Tang, B., and K. Emanuel, 2010: Midlevel ventilation's constraint on tropical cyclone intensity. J. Atmos. Sci., 67, 1817-1830, doi:10.1175/2010JAS3318.1.

— , and — 2012: Sensitivity of tropical cyclone intensity to ventilation in an axisymmetric model. J. Atmos. Sci., 69, 23942413, doi:10.1175/JAS-D-11-0232.1.

Vecchi, G. A., and B. J. Soden, 2007: Effect of remote sea surface temperature change on tropical cyclone potential intensity. Nature, 450, 1066-1071, doi:10.1038/nature06423.

_ S. Fueglistaler, I. M. Held, T. R. Knutson, and M. Zhao, 2013: Impacts of atmospheric temperature trends on tropical cyclone activity. J. Climate, 26,3877-3891, doi:10.1175/JCLI-D-12-00503.1.

Wang, S., and A. H. Sobel, 2011: Response of convection to relative sea surface temperature: Cloud-resolving simulations in two and three dimensions. J. Geophys. Res., 116, D11119, doi:10.1029/ $2010 J D 015347$.

Wang, Y., and J. Xu, 2010: Energy production, frictional dissipation, and maximum intensity of a numerically simulated tropical cyclone. J. Atmos. Sci., 67, 97-116, doi:10.1175/2009JAS3143.1.

Wing, A. A., A. H. Sobel, and S. J. Camargo, 2007: Relationship between the potential and actual intensities of tropical cyclones on interannual time scales. Geophys. Res. Lett., 34, L08810, doi:10.1029/2006GL028581.

Yang, B., Y. Wang, and B. Wang, 2007: The effect of internally generated inner-core asymmetries on tropical cyclone potential intensity. J. Atmos. Sci., 64, 1165-1188, doi:10.1175/JAS3971.1.

Zhang, D.-L., and E. Altshuler, 1999: The effects of dissipative heating on hurricane intensity. Mon. Wea. Rev., 127,3032-3038, doi:10.1175/1520-0493(1999)127<3032:TEODHO>2.0.CO;2.

Zhang, J. A., 2010: Estimation of dissipative heating using lowlevel in situ aircraft observations in the hurricane boundary layer. J. Atmos. Sci., 67, 1853-1862, doi:10.1175/2010JAS3397.1.

— , P. Zhu, F. J. Masters, R. F. Rogers, and F. D. Marks, 2011: On momentum transport and dissipative heating during hurricane landfalls. J. Atmos. Sci., 68, 1397-1404, doi:10.1175/ JAS-D-10-05018.1.

Zhao, M., and I. M. Held, 2012: TC-permitting GCM simulations of hurricane frequency response to sea surface temperature anomalies projected for the late-twenty-first century. J. Climate, 25, 2995-3009, doi:10.1175/JCLI-D-11-00313.1.

,-- S.-J. Lin, and G. A. Vecchi, 2009: Simulations of global hurricane climatology, interannual variability, and response to global warming using a 50-21 km resolution GCM. J. Climate, 22, 6653-6678, doi:10.1175/2009JCLI3049.1. 